\title{
Multivariate Selection Index of Acerola Genotypes for Fresh Consumption Based on Fruit Physicochemical Attributes
}

\section{Maria A.R. Ferreira}

EMBRAPA Centro de Pesquisa Agropecuária do Trópico Semiárido: EMBRAPA Centro de Pesquisa Agropecuaria do Tropico Semiarido

João Claudio Vilvert

EMBRAPA Centro de Pesquisa Agropecuária do Trópico Semiárido: EMBRAPA Centro de Pesquisa Agropecuaria do Tropico Semiarido

Bárbara 0.S. Silva

EMBRAPA Centro de Pesquisa Agropecuária do Trópico Semiárido: EMBRAPA Centro de Pesquisa Agropecuaria do Tropico Semiarido

lanca Carneiro Ferreira

Federal University of Lavras: Universidade Federal de Lavras

Flávio França Souza

EMBRAPA Centro de Pesquisa Agropecuária do Trópico Semiárido: EMBRAPA Centro de Pesquisa Agropecuaria do Tropico Semiarido

\section{Sergio Tonetto De Freitas ( $\nabla$ sergio.freitas@embrapa.br)}

EMBRAPA Centro de Pesquisa Agropecuária do Trópico Semiárido: EMBRAPA Centro de Pesquisa Agropecuaria do Tropico Semiarido https://orcid.org/0000-0001-9579-7304

\section{Research Article}

Keywords: Malpighia emarginata DC., Ascorbic acid, Quality, Descriptors, Genetic diversity, Consumers

Posted Date: June 28th, 2021

DOI: https://doi.org/10.21203/rs.3.rs-643152/v1

License: (1) This work is licensed under a Creative Commons Attribution 4.0 International License. Read Full License

Version of Record: A version of this preprint was published at Euphytica on February 9th, 2022. See the published version at https://doi.org/10.1007/s10681-022-02978-1. 


\section{Abstract}

Acerola (Malpighia emarginata DC.) is one of the most important fruit produced in the São Francisco Valley, a Semi-arid region in the Northeast of Brazil. This study aimed to evaluate the physicochemical quality of thirty-five acerola genotypes produced during two growing seasons in a Semi-arid region and to identify the best ones with potential for fresh consumption based on a multivariate selection index. Fruit of each genotype were harvested during two growing seasons at the maturity stage red-ripe, characterized by full red skin color. After harvest, the fruit were evaluated for diameter, mass, flesh firmness, soluble solids (SS), titratable acidity (TA), SS/TA ratio, ascorbic acid content and skin color. A multivariate selection index (SI) was applied for scoring and ranking the genotypes for fresh consumption based on red-ripe fruit physicochemical quality. According to the results, all physicochemical attributes had high variability among genotypes. The SI was a powerful tool for identifying genotypes with high potential for fresh consumption, since it allowed selecting genotypes with multiple desirable traits. In the first and second growing seasons, the SI identified the genotypes PROG $052(\mathrm{SI}=76.1$ and 78.9), BRS Rubra $(\mathrm{SI}=74.1$ and 99.5), Cabocla $(\mathrm{SI}=72.3$ and 70.7), Costa Rica (SI = 61.2 and 73.8) and PROG 069 (SI = 68.1 and 72.4) as the most promising ones for fresh consumption due to the presence of multiple desirable traits such as high diameter, mass, flesh firmness, SS, and SS/AT ratio, as well as lower acidity.

\section{Introduction}

Acerola (Malpighia emarginata DC.) is a tropical super-fruit due to its high vitamin C content that exceeds 100 times the contents observed in oranges and lemons (Prakash and Baskaran, 2018). The worldwide cultivation of acerola takes place from South Texas, Mexico, Central America and Northern regions in the South America, as well as some regions of Southeast Asia, specially India (Assis et al., 2008; Hanamura et al., 2008). Currently, Brazil is the largest producer of acerola, with several cultivation areas in the Northeastern region, where the environmental conditions enable three to four harvests per year (Alves et al., 1999).

The production of acerolas in Brazil is intended for the fresh fruit market and/or processing industry. Genotypes intended for fresh consumption are characterized by larger and firmer fruit, with higher soluble solids content and lower levels of organic acids, which guarantees the sweetness desired by consumers (Hoehn et al., 2005). On the other hand, genotypes intended for the processing industry must have higher levels of ascorbic acid, which is one of the main forms of processed products. Acerola can also be marketed as frozen pulp, concentrate, juice, ice cream, gelatin, soft drinks, nectar, jelly, gum, preserve, yogurts and sodas (Delva and Schneider, 2013; Mezadri et al., 2006).

The quality of each acerola genotype is highly dependent on environmental conditions such as temperature, precipitation and sunlight, as well as on crop management practices such as irrigation, fertilization, pest and disease control, maturity stage at harvest and storage conditions (Alves et al., 1999; Delva and Schneider, 2013). In addition, the species has high genetic variability that leads to high diversity of fruit quality due to its wide distribution and cultivation (Assis et al., 2008; Hanamura et al., 2008; Ritzinger et al., 2017). Although small scale cultivation has been carried out for over 50 years in Brazil, commercial cultivation of acerola is quite recent and has been accomplished mainly with a few cultivars such as Junko, Flor Branca, Costa Rica, BRS Sertaneja, Okinawa, Nikki, Coopama, and BRS Cabocla (Assis et al., 2008; Souza, 2015). The acerola germplasm bank (AGB) at the Tropical Semi-arid Embrapa, Petrolina, PE, Brazil was established in 2012 and contains acerola genotypes from different regions in the States of Pernambuco, Ceará, Bahia, Paraíba, Paraná and São Paulo in Brazil. Fruit quality of these genotypes has to be evaluated in order to select the ones with the highest potential for the fresh market and for breeding programs to obtain new genotypes with higher quality. These studies will help improving acerola quality in the market, stimulating consumption, production and increasing the income for smallholder farmers that are the main acerola growers in the country. In this context, the rank-summation index method has been shown to be an efficient approach to select genotypes based on multiple desirable traits, which can be used to rank genotypes with combined quality traits for fresh fruit consumption and breeding programs (Mulamba and Mock, 1978; Bertini et al., 2010; Poggetti et al., 2017; Barth et al., 2020).

This study aimed to evaluate the physicochemical quality of thirty-five acerola genotypes produced during two growing seasons in a Semi-arid region and to identify the best ones with potential for fresh consumption based on a multivariate selection index.

\section{Material And Methods}

\subsection{Acerola genotypes and environmental conditions}


The study was carried out in the acerola germplasm bank (AGB) at the Tropical Semi-arid Embrapa, Petrolina, PE, Brazil $\left(09^{\circ} 09^{\prime} \mathrm{S}\right.$, $40^{\circ} 22^{\prime} \mathrm{W}$ and $365 \mathrm{~m}$ above the sea level), during two growing seasons in 2019 and 2020. The region has a Semi-arid climate (Bswh according to Koppen) with average annual temperature of $26^{\circ} \mathrm{C}$, rainfall of $500 \mathrm{~mm}$, and relative humidity of $66 \%$. The acerola plants were daily irrigated, and the amount of water applied was determined based on crop evapotranspiration. Fertilization and phytosanitary treatment were carried out according to technical recommendations (Ritzinger et al., 2003).

Thirty-five acerola genotypes were harvested when the fruit reached the red-ripe maturity stage, characterized by the full red color of the skin (Fig. 1). Parentage and origin of the genotypes are shown in Supplementary Table S1. The study followed a randomized complete block design, where each genotype was represented by three blocks and each block by four plants. Harvest was accomplished early in the morning and the fruit were immediately transported to the Postharvest Laboratory at Tropical Semi-Arid Embrapa, Petrolina, PE, Brazil. The distance between the experimental field and the laboratory is about $10 \mathrm{~km}$. A total of 10 healthy fruit per block were subjected to physicochemical analysis as described below.

\subsection{Physicochemical analyses}

Fruit diameter was determined individually with a digital caliper Mitutoyo (model CD-6 CS, Mitutoyo Corp., Japan). Diameter results were presented in centimeter. Fruit fresh mass was individually determined with a digital balance AD50 (Marte Científica, Brazil). Fresh mass results were presented in gram. Fruit firmness was determined as the maximum force required to press $10 \%$ of the fruit diameter using a TA.XT.Plus Texture Anlyzer (Extralab ${ }^{\circledR}$, Brazil), adapted with a P/75 pressure plate. Flesh firmness results were expressed in Newton (N).

Soluble solids (SS) were determined in juice samples using a digital refractometer PAL-1 (Atago, Brazil) with automatic temperature compensation. The results were expressed in percentage. Titratable acidity (TA) was evaluated by titration of $1 \mathrm{~mL}$ of juice diluted in $50 \mathrm{~mL}$ of distilled water with a solution of $0.1 \mathrm{~N} \mathrm{NaOH}$ until pH 8.1. Results were expressed as percentage of malic acid in acerola juice.

Ascorbic acid (AA) content was quantified by titration with Tillman's solution (DFI - 2,6-dichlorophenol indophenol) at $0.02 \%$. A total of $1.0 \mathrm{~mL}$ of acerola juice was diluted in $100 \mathrm{ml}$ of oxalic acid at $0.5 \%$. Later, $1 \mathrm{~mL}$ of this solution was diluted in $50 \mathrm{~mL}$ of distilled water and titrated with Tillman's solution until permanent light pink color development (Strohecker and Henning, 1967). Results were expressed as percentage of AA in acerola juice.

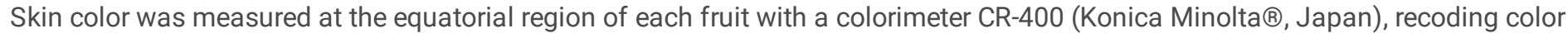
measurements in the CIELab system, where L* represents the lightness, $C$ represents the chroma, and $\mathrm{h}$ represents the hue angle.

\subsection{Statistical analysis}

The selection of superior acerola genotypes with combined quality traits desirable for fresh consumption was accomplished with physicochemical data obtained from red-ripe fruit. The most important quality traits considered for genotype selection were fruit diameter, mass, flesh firmness, SS, TA, SS/TA ratio and AA. Skin color parameters were not considered for genotype selection.

A multivariate selection index for scoring and ranking the genotypes was applied according to previous studies (Mulamba and Mock, 1978; Poggetti et al., 2017; Barth et al., 2020). The multivariate selection index was calculated based on the seven quality trait variables (diameter, mass, flesh firmness, SS, TA, SS/TA, AA), which were standardized and multiplied by the weighting coefficient (Kx) adopted for each variable (Table 1). These weighing coefficients were determined for each variable, following a previous study that applied the same selection index for physicochemical traits of strawberry genotypes intended for fresh consumption (Barth et al., 2020). Therefore, each variable was standardized to the unit, so that each variable had the same relative weight, and multiplied by the weighting coefficient $(\mathrm{Kx})$. The formula of the selection index (SI) was: SI $=\mathrm{K} 1$ * ((diameter-min1)/R1) + K2 * ((mass-min2)/R2) + K3 * $((\mathrm{firmness}-\mathrm{min} 3) / \mathrm{R} 3)+\mathrm{K} 4$ * ((SS-min4)/R4) + K5 * ((TA-min5)/R5) + K6 * ((SS/TA-min6)/R6) + K7 * ((AA-min7)/R7), where: K1, K2, K3, $\mathrm{K} 4, \mathrm{~K} 5, \mathrm{~K} 6, \mathrm{~K} 7$ are the weighting coefficient assigned to the variables (Table 1); $\min 1, \min 2, \min 3, \min 4, \min 5, \min 6, \min 7$ are the minimum values assumed by the variables (Table 2); R1, R2, R3, R4, R5, R6, R7 are the ranges of variation of the variables (Table 2). For instance, the calculation of the selection index for the genotype PROG 052 in the first growing season is shown below.

SI for diameter $=20 *[(2.79-1.51) / 1.28]=20.0$

SI for mass $=15 *[(10.80-1.81) / 8.99]=15.0$ 
SI for flesh firmness $=20 *[(17.38-5.02) / 15.00]=16.5$

SI for SS $=15 *[(8.00-6.03) / 7.04]=4.2$

SI for TA $=15-15 *[(1.49-0.87) / 1.10]=6.5$

SI for $S S / T A=20 *[(5.39-4.06) / 6.75]=3.9$

SI for $A A=15-15 *[(1.48-0.81) / 1.99]=9.9$

Final SI $(\Sigma$ SIs $)=76.1$

The genotype PROG 052 had the maximum ranks for diameter (20.0) and mass (15.0), because it had the highest values for these traits among the genotypes. The ranks for TA and AA were inverted, so that the higher TA and AA, the lower the rank, because higher quality for consumption requires lower acid taste of the fruit.

The physicochemical attributes were subjected to the Analysis of Variance (ANOVA). Mean values were compared between growing seasons by the F-test. Mean values were compared among genotypes, in each growing season, by the Scott-Knott test (5\%). Principal component analysis (PCA) was applied to the variables to reduce the data structure and to investigate distribution of acerola genotypes on factor plots (PC1 versus PC2 and PC1 vs PC3). Statistical analyses were performed using Minitab software version 19 (Minitab Inc., USA).

Table 1

Weighting coefficient $(K)$ adopted for physicochemical attributes of acerola fruit used to select the most promising acerola genotypes for fresh consumption.

\begin{tabular}{|l|l|}
\hline Quality trait & K \\
\hline Diameter & 20 \\
\hline Mass & 15 \\
\hline Firmness & 20 \\
\hline SS & 15 \\
\hline TA* & 15 \\
\hline SS/TA ratio & 20 \\
\hline AA* & 15 \\
\hline$*$ For all traits, higher values are preferable, with the exception of TA and AA, whose lower values are desirable. \\
\hline
\end{tabular}


Table 2

Physicochemical quality of 35 red-ripe acerola genotypes harvested in 2019 and 2020 in the São Francisco Valley, Petrolina, PE, Brazil.

\begin{tabular}{|c|c|c|c|c|c|c|}
\hline \multirow[t]{2}{*}{ Quality Trait } & \multicolumn{2}{|c|}{ Harvest in 2019} & \multicolumn{2}{|c|}{ Harvest in 2020} & \multirow[t]{2}{*}{$F$} & \multirow[t]{2}{*}{ CV (\%) } \\
\hline & Average $^{a}$ & Range & Average & Range & & \\
\hline Diameter (cm) & 2.15 & $1.51-2.79$ & 2.15 & $1.74-2.50$ & $0.00^{\mathrm{ns}}$ & 6.21 \\
\hline Mass (g) & 5.23 & $1.81-10.80$ & 5.12 & $2.86-8.00$ & $1.38^{\mathrm{ns}}$ & 13.23 \\
\hline Firmness (N) & 10.11 & $5.02-20.02$ & 11.32 & $6.95-18.75$ & $31.08^{*}$ & 14.43 \\
\hline SS (\%) & 8.82 & $6.03-13.07$ & 10.50 & $7.50-13.87$ & $149.69^{\star}$ & 8.05 \\
\hline TA (\%) & 1.39 & $0.87-1.97$ & 1.40 & $0.79-1.89$ & $0.00^{\mathrm{ns}}$ & 9.72 \\
\hline SS/TA & 6.55 & $4.06-10.81$ & 7.93 & $4.91-15.84$ & $161.55^{\star \star}$ & 10.82 \\
\hline AA (\%) & 1.56 & $0.81-2.80$ & 1.56 & $0.81-2.72$ & $0.03^{\mathrm{ns}}$ & 16.86 \\
\hline Lightness & 39.40 & $32.67-44.15$ & 38.62 & $32.47-51.83$ & $1.56^{\mathrm{ns}}$ & 7.50 \\
\hline Chroma & 43.98 & $30.84-53.29$ & 42.05 & $27.66-52.59$ & $12.40^{\star}$ & 7.46 \\
\hline Hue angle & 28.75 & $19.45-36.43$ & 28.09 & $20.50-45.82$ & $1.47^{\mathrm{ns}}$ & 10.09 \\
\hline
\end{tabular}

\section{Results}

Physicochemical quality of acerola genotypes harvested at the red-ripe maturity stage in 2019 and 2020 growing seasons is presented in Tables 2, 3 and 4. According to the results, the growing season showed a significant effect only on flesh firmness, skin chroma, SS, and SS/TA ratio of acerola fruit (Table 2). The genotypes showed statistical difference for all quality traits in both growing seasons (Tables 3 and 4). 
Table 3

Physicochemical attributes of red-ripe fruit and ranks of acerola genotypes based on the multivariate selection index used to identify the most promising genotypes for fresh fruit consumption. All genotypes were harvested in the 2019 growing season, in the São

Francisco Valley, Petrolina, PE, Brazil.

\begin{tabular}{|c|c|c|c|c|c|c|c|c|c|c|c|c|}
\hline & Genotype & $\begin{array}{l}\text { Diameter } \\
\text { (cm) }\end{array}$ & $\begin{array}{l}\text { Mass } \\
(\mathrm{g})\end{array}$ & $\begin{array}{l}\mathrm{FF} \\
(\mathrm{N})\end{array}$ & $\begin{array}{l}\text { SS } \\
(\%)\end{array}$ & $\begin{array}{l}\text { TA } \\
(\%)\end{array}$ & SS/TA & $\begin{array}{l}\text { AA } \\
(\%)\end{array}$ & Lightness & Chroma & $\begin{array}{l}\text { Hue } \\
\text { angle }\end{array}$ & Ranking \\
\hline $1 \mathrm{st}$ & $\begin{array}{l}\text { PROG } \\
052\end{array}$ & $2.79 a^{*}$ & $\begin{array}{l}10.80 \\
\mathrm{a}\end{array}$ & $\begin{array}{l}17.38 \\
b\end{array}$ & $\begin{array}{l}8.00 \\
d\end{array}$ & $\begin{array}{l}1.49 \\
b\end{array}$ & $5.39 \mathrm{~d}$ & $\begin{array}{l}1.48 \\
d\end{array}$ & $35.92 \mathrm{~b}$ & $41.11 \mathrm{c}$ & $\begin{array}{l}36.43 \\
a\end{array}$ & 76.11 \\
\hline 2nd & $\begin{array}{l}\text { BRS } \\
\text { Rubra }\end{array}$ & $2.24 \mathrm{c}$ & $\begin{array}{l}4.95 \\
d\end{array}$ & $\begin{array}{l}8.48 \\
d\end{array}$ & $\begin{array}{l}9.33 \\
c\end{array}$ & $\begin{array}{l}0.93 \\
d\end{array}$ & $\begin{array}{l}10.08 \\
a\end{array}$ & $\begin{array}{l}0.97 \\
\mathrm{e}^{-9}\end{array}$ & $43.65 \mathrm{a}$ & $47.07 \mathrm{~b}$ & $\begin{array}{l}31.86 \\
a\end{array}$ & 74.10 \\
\hline $3 r d$ & Cabocla & $2.42 \mathrm{c}$ & $\begin{array}{l}6.51 \\
c\end{array}$ & $\begin{array}{l}10.20 \\
d\end{array}$ & $\begin{array}{l}10.27 \\
\mathrm{C}\end{array}$ & $\begin{array}{l}1.18 \\
\mathrm{c}\end{array}$ & 8.78 b & $\begin{array}{l}1.52 \\
d\end{array}$ & 42.49 a & $52.53 \mathrm{a}$ & $\begin{array}{l}30.35 \\
\text { a }\end{array}$ & 72.37 \\
\hline 4th & $\begin{array}{l}\text { PROG } \\
069\end{array}$ & $2.34 \mathrm{c}$ & $\begin{array}{l}5.54 \\
d\end{array}$ & $\begin{array}{l}20.02 \\
a\end{array}$ & $\begin{array}{l}7.87 \\
d\end{array}$ & $\begin{array}{l}1.32 \\
b\end{array}$ & $6.04 \mathrm{c}$ & $\begin{array}{l}1.43 \\
d\end{array}$ & $35.06 \mathrm{~b}$ & $36.23 d$ & $\begin{array}{l}23.25 \\
c\end{array}$ & 68.17 \\
\hline 5th & CARP 01 & $2.51 \mathrm{~b}$ & $\begin{array}{l}8.07 \\
b\end{array}$ & $\begin{array}{l}8.18 \\
\mathrm{e}\end{array}$ & $\begin{array}{l}7.50 \\
\mathrm{e}\end{array}$ & $\begin{array}{l}1.11 \\
\mathrm{C}\end{array}$ & $6.77 c$ & $\begin{array}{l}1.02 \\
e^{-02}\end{array}$ & $39.23 \mathrm{a}$ & $44.57 \mathrm{~b}$ & $\begin{array}{l}28.89 \\
a\end{array}$ & 66.59 \\
\hline 6th & Olivier & $1.93 \mathrm{~d}$ & $\begin{array}{l}4.08 \\
e\end{array}$ & $\begin{array}{l}7.48 \\
\mathrm{e}\end{array}$ & $\begin{array}{l}8.93 \\
d\end{array}$ & $\begin{array}{l}0.92 \\
d\end{array}$ & 9.73 a & $\begin{array}{l}0.82 \\
\mathrm{e}\end{array}$ & $36.39 \mathrm{~b}$ & $39.28 \mathrm{c}$ & $\begin{array}{l}26.00 \\
b\end{array}$ & 65.85 \\
\hline 7th & $\begin{array}{l}\text { PROG } \\
233\end{array}$ & $1.97 d$ & $\begin{array}{l}4.06 \\
e\end{array}$ & $\begin{array}{l}5.14 \\
e^{-14}\end{array}$ & $\begin{array}{l}11.27 \\
b\end{array}$ & $\begin{array}{l}1.06 \\
c\end{array}$ & $\begin{array}{l}10.81 \\
a\end{array}$ & $\frac{1.33}{d}$ & $40.52 \mathrm{a}$ & $46.67 \mathrm{~b}$ & $\begin{array}{l}31.09 \\
a\end{array}$ & 65.76 \\
\hline 8th & UEL 03 & $2.31 \mathrm{c}$ & $\begin{array}{l}7.08 \\
c\end{array}$ & $\begin{array}{l}13.49 \\
c\end{array}$ & $\begin{array}{l}9.20 \\
c\end{array}$ & $\begin{array}{l}1.28 \\
c\end{array}$ & $7.20 \mathrm{c}$ & $\begin{array}{l}1.87 \\
C\end{array}$ & $38.48 \mathrm{a}$ & $44.20 \mathrm{~b}$ & $\begin{array}{l}30.36 \\
a\end{array}$ & 65.06 \\
\hline 9th & $\begin{array}{l}\text { PROG } \\
123\end{array}$ & $2.23 \mathrm{c}$ & $\begin{array}{l}5.90 \\
\mathrm{C}\end{array}$ & $\begin{array}{l}9.39 \\
d\end{array}$ & $e^{7.47}$ & $\begin{array}{l}0.97 \\
d\end{array}$ & $7.69 \mathrm{~b}$ & $\begin{array}{l}1.36 \\
d\end{array}$ & $39.48 \mathrm{a}$ & $42.24 \mathrm{c}$ & $\begin{array}{l}30.46 \\
a\end{array}$ & 62.22 \\
\hline 10th & $\begin{array}{l}\text { Costa } \\
\text { Rica }\end{array}$ & $2.30 \mathrm{c}$ & $\begin{array}{l}5.59 \\
d\end{array}$ & $\begin{array}{l}6.42 \\
\mathrm{e}\end{array}$ & $\begin{array}{l}11.43 \\
b\end{array}$ & $\begin{array}{l}1.43 \\
b\end{array}$ & $8.08 \mathrm{~b}$ & $\begin{array}{l}1.48 \\
d\end{array}$ & $43.09 \mathrm{a}$ & $50.73 \mathrm{a}$ & $\begin{array}{l}32.57 \\
a\end{array}$ & 61.25 \\
\hline 11th & $\begin{array}{l}\text { PROG } \\
215\end{array}$ & $2.18 \mathrm{c}$ & $\begin{array}{l}5.43 \\
d\end{array}$ & $\begin{array}{l}7.60 \\
e^{-1}\end{array}$ & $e^{6.63}$ & $\begin{array}{l}0.87 \\
d\end{array}$ & 7.66 b & $\mathrm{e}^{1.02}$ & $41.57 \mathrm{a}$ & $48.38 \mathrm{~b}$ & $\begin{array}{l}30.72 \\
a\end{array}$ & 60.31 \\
\hline 12th & ACO 17 & $2.13 \mathrm{c}$ & $\begin{array}{l}5.07 \\
d\end{array}$ & $\begin{array}{l}11.12 \\
d\end{array}$ & $\begin{array}{l}10.70 \\
\text { b }\end{array}$ & $\begin{array}{l}1.48 \\
b\end{array}$ & $7.29 \mathrm{c}$ & $\begin{array}{l}1.55 \\
d\end{array}$ & $\star \star$ & $\star \star$ & $\star \star$ & 58.88 \\
\hline 13th & $\begin{array}{l}\text { PROG } \\
081\end{array}$ & $2.59 \mathrm{~b}$ & $\begin{array}{l}8.97 \\
b\end{array}$ & $\begin{array}{l}12.46 \\
c\end{array}$ & $e^{6.03}$ & $\begin{array}{l}1.39 \\
b\end{array}$ & $4.34 \mathrm{~d}$ & 1.45 & $\star *$ & $\star *$ & $\star \star$ & 57.66 \\
\hline 14th & ACO 18 & $2.10 \mathrm{~d}$ & $\begin{array}{l}5.49 \\
d\end{array}$ & $\begin{array}{l}12.90 \\
\mathrm{c}\end{array}$ & $\begin{array}{l}6.60 \\
\mathrm{e}\end{array}$ & $\begin{array}{l}1.11 \\
\mathrm{c}\end{array}$ & $5.95 c$ & $\begin{array}{l}1.20 \\
d\end{array}$ & $42.45 \mathrm{a}$ & $39.00 \mathrm{c}$ & $\begin{array}{l}32.33 \\
a\end{array}$ & 56.47 \\
\hline 15th & ACO 10 & $1.85 \mathrm{~d}$ & $\begin{array}{l}3.69 \\
f\end{array}$ & $\begin{array}{l}16.16 \\
b\end{array}$ & $\begin{array}{l}11.20 \\
b\end{array}$ & $\begin{array}{l}1.66 \\
a\end{array}$ & $6.93 c$ & $\begin{array}{l}1.60 \\
d\end{array}$ & $39.33 \mathrm{a}$ & $43.30 \mathrm{c}$ & $\begin{array}{l}29.36 \\
a\end{array}$ & 56.09 \\
\hline 16th & RECI 02 & $2.38 \mathrm{c}$ & $\begin{array}{l}6.83 \\
c\end{array}$ & $\begin{array}{l}9.45 \\
d\end{array}$ & 8.80 & $\begin{array}{l}1.46 \\
b\end{array}$ & $6.03 c$ & $\begin{array}{l}1.57 \\
d\end{array}$ & $41.02 \mathrm{a}$ & $45.87 \mathrm{~b}$ & $\begin{array}{l}32.40 \\
a\end{array}$ & 55.84 \\
\hline 17th & $\begin{array}{l}\text { PROG } \\
023\end{array}$ & $2.09 \mathrm{~d}$ & $\begin{array}{l}4.74 \\
d\end{array}$ & $\begin{array}{l}14.21 \\
c\end{array}$ & $\begin{array}{l}10.30 \\
c\end{array}$ & $\begin{array}{l}1.52 \\
b\end{array}$ & $6.75 c$ & $\begin{array}{l}1.97 \\
C\end{array}$ & $33.44 \mathrm{~b}$ & $32.64 \mathrm{~d}$ & $\begin{array}{l}19.45 \\
C\end{array}$ & 55.67 \\
\hline 18th & $\begin{array}{l}\text { PROG } \\
195\end{array}$ & $2.16 \mathrm{c}$ & $\begin{array}{l}5.20 \\
d\end{array}$ & $\begin{array}{l}10.23 \\
d\end{array}$ & $\begin{array}{l}7.67 \\
d\end{array}$ & $\begin{array}{l}1.18 \\
\mathrm{c}\end{array}$ & $6.51 \mathrm{c}$ & $\begin{array}{l}1.42 \\
d\end{array}$ & $38.41 \mathrm{a}$ & $42.97 \mathrm{c}$ & $\begin{array}{l}31.05 \\
a\end{array}$ & 54.69 \\
\hline 19th & $\begin{array}{l}\text { PROG } \\
122\end{array}$ & $2.27 \mathrm{c}$ & $\begin{array}{l}6.03 \\
c\end{array}$ & $\begin{array}{l}9.41 \\
d\end{array}$ & $\begin{array}{l}7.93 \\
d\end{array}$ & $\begin{array}{l}1.41 \\
b\end{array}$ & $5.67 \mathrm{~d}$ & $\begin{array}{l}1.32 \\
d\end{array}$ & $32.67 \mathrm{~b}$ & $30.84 \mathrm{~d}$ & $\begin{array}{l}21.13 \\
c\end{array}$ & 52.38 \\
\hline 20th & Okinawa & $2.35 \mathrm{c}$ & $\begin{array}{l}7.07 \\
\mathrm{C}\end{array}$ & $\begin{array}{l}10.66 \\
d\end{array}$ & $\begin{array}{l}10.70 \\
b\end{array}$ & $\begin{array}{l}1.78 \\
a\end{array}$ & $6.01 \mathrm{c}$ & $\begin{array}{l}2.20 \\
b\end{array}$ & 39.87 a & $43.10 \mathrm{c}$ & $\begin{array}{l}28.56 \\
a\end{array}$ & 52.26 \\
\hline $21 \mathrm{st}$ & BV 07 & $2.03 d$ & $\begin{array}{l}4.20 \\
\mathrm{e}\end{array}$ & $\begin{array}{l}7.10 \\
\mathrm{e}\end{array}$ & $\begin{array}{l}7.27 \\
\mathrm{e}\end{array}$ & $\begin{array}{l}1.12 \\
\mathrm{C}\end{array}$ & $6.52 c$ & $\begin{array}{l}0.81 \\
\mathrm{e}\end{array}$ & $44.15 \mathrm{a}$ & $53.29 a$ & $\begin{array}{l}32.50 \\
a\end{array}$ & 51.41 \\
\hline
\end{tabular}




\begin{tabular}{|c|c|c|c|c|c|c|c|c|c|c|c|c|}
\hline & Genotype & $\begin{array}{l}\text { Diameter } \\
\text { (cm) }\end{array}$ & $\begin{array}{l}\text { Mass } \\
\text { (g) }\end{array}$ & $\begin{array}{l}\mathrm{FF} \\
(\mathrm{N})\end{array}$ & $\begin{array}{l}\text { SS } \\
(\%)\end{array}$ & $\begin{array}{l}\text { TA } \\
(\%)\end{array}$ & SS/TA & $\begin{array}{l}\text { AA } \\
(\%)\end{array}$ & Lightness & Chroma & $\begin{array}{l}\text { Hue } \\
\text { angle }\end{array}$ & Ranking \\
\hline 22nd & ACO 35 & $1.80 \mathrm{~d}$ & $\begin{array}{l}2.60 \\
\mathrm{~g}\end{array}$ & $\begin{array}{l}8.03 \\
\mathrm{e}\end{array}$ & $\begin{array}{l}13.07 \\
a\end{array}$ & $\begin{array}{l}1.66 \\
a\end{array}$ & $7.96 \mathrm{~b}$ & $\begin{array}{l}1.64 \\
\mathrm{C}\end{array}$ & $37.70 \mathrm{a}$ & $44.74 \mathrm{~b}$ & $\begin{array}{l}25.47 \\
b\end{array}$ & 49.39 \\
\hline $23 r d$ & ALHA 06 & $2.31 \mathrm{c}$ & $\begin{array}{l}6.68 \\
c\end{array}$ & $\begin{array}{l}9.67 \\
d\end{array}$ & $\begin{array}{l}9.43 \\
c\end{array}$ & $\begin{array}{l}1.78 \\
a\end{array}$ & $5.30 \mathrm{~d}$ & $\begin{array}{l}1.74 \\
\mathrm{c}\end{array}$ & $40.94 \mathrm{a}$ & $32.71 \mathrm{~d}$ & $\begin{array}{l}27.88 \\
a\end{array}$ & 48.32 \\
\hline 24th & $\begin{array}{l}\text { PROG } \\
188\end{array}$ & $2.31 \mathrm{c}$ & $\begin{array}{l}6.17 \\
c\end{array}$ & $\begin{array}{l}10.71 \\
d\end{array}$ & $\begin{array}{l}9.40 \\
\mathrm{C}\end{array}$ & $\begin{array}{l}1.71 \\
a\end{array}$ & $5.51 \mathrm{~d}$ & $\begin{array}{l}2.03 \\
\mathrm{C}\end{array}$ & 37.31 b & $38.25 \mathrm{~d}$ & $\begin{array}{l}26.22 \\
b\end{array}$ & 48.19 \\
\hline 25th & ACO 03 & $2.21 \mathrm{c}$ & $e^{4.40}$ & $e^{7.16}$ & $\begin{array}{l}8.97 \\
d\end{array}$ & $\begin{array}{l}1.53 \\
b\end{array}$ & $5.89 \mathrm{c}$ & $\begin{array}{l}1.25 \\
d\end{array}$ & $42.84 \mathrm{a}$ & $52.80 \mathrm{a}$ & $\begin{array}{l}33.08 \\
a\end{array}$ & 47.48 \\
\hline 26th & $\begin{array}{l}\text { PROG } \\
216\end{array}$ & $1.86 \mathrm{~d}$ & $\begin{array}{l}3.72 \\
f\end{array}$ & $\begin{array}{l}11.63 \\
c\end{array}$ & $\begin{array}{l}11.13 \\
b\end{array}$ & $\begin{array}{l}1.68 \\
a\end{array}$ & $6.65 c$ & $\begin{array}{l}1.92 \\
\mathrm{c}\end{array}$ & $37.39 \mathrm{~b}$ & $46.79 \mathrm{~b}$ & $\begin{array}{l}25.66 \\
b\end{array}$ & 46.60 \\
\hline 27th & Valéria & $2.33 \mathrm{c}$ & $\begin{array}{l}6.28 \\
c\end{array}$ & $\begin{array}{l}6.06 \\
\mathrm{e}\end{array}$ & $\begin{array}{l}8.13 \\
d\end{array}$ & $\begin{array}{l}1.53 \\
b\end{array}$ & $5.36 \mathrm{~d}$ & $\begin{array}{l}1.41 \\
d\end{array}$ & $34.66 \mathrm{~b}$ & $40.12 \mathrm{c}$ & $\begin{array}{l}21.68 \\
c\end{array}$ & 46.46 \\
\hline 28th & $\begin{array}{l}\text { PROG } \\
102\end{array}$ & $2.16 \mathrm{c}$ & $\begin{array}{l}2.16 \\
\mathrm{~g}\end{array}$ & $\begin{array}{l}10.16 \\
d\end{array}$ & $\begin{array}{l}8.50 \\
d\end{array}$ & $\begin{array}{l}1.39 \\
b\end{array}$ & $6.14 \mathrm{c}$ & $\begin{array}{l}1.70 \\
\mathrm{c}\end{array}$ & $34.29 b$ & $39.76 \mathrm{c}$ & $\begin{array}{l}21.49 \\
c\end{array}$ & 45.22 \\
\hline 29th & Barbados & $1.96 \mathrm{~d}$ & $\begin{array}{l}3.37 \\
f\end{array}$ & $e^{6.46}$ & $e^{6.67}$ & $\begin{array}{l}1.15 \\
c\end{array}$ & $5.79 \mathrm{~d}$ & $\begin{array}{l}1.20 \\
d\end{array}$ & $39.17 \mathrm{a}$ & $42.50 \mathrm{c}$ & $\begin{array}{l}24.69 \\
b\end{array}$ & 41.29 \\
\hline 30th & $\begin{array}{l}\text { PROG } \\
244\end{array}$ & $2.04 \mathrm{~d}$ & $e^{4.19}$ & $\begin{array}{l}13.37 \\
c\end{array}$ & $\begin{array}{l}7.47 \\
e^{-1}\end{array}$ & $\begin{array}{l}1.65 \\
a\end{array}$ & $4.52 \mathrm{~d}$ & $\begin{array}{l}1.88 \\
\mathrm{C}\end{array}$ & $41.90 \mathrm{a}$ & $49.92 \mathrm{a}$ & $\begin{array}{l}33.17 \\
a\end{array}$ & 39.12 \\
\hline $31 \mathrm{st}$ & $\begin{array}{l}\text { PROG } \\
046\end{array}$ & $1.93 \mathrm{~d}$ & $\begin{array}{l}3.93 \\
e^{-1}\end{array}$ & $\begin{array}{l}9.27 \\
d\end{array}$ & $\begin{array}{l}7.07 \\
\mathrm{e}\end{array}$ & $\begin{array}{l}1.33 \\
b\end{array}$ & $5.32 \mathrm{~d}$ & $\begin{array}{l}1.70 \\
c\end{array}$ & $43.83 \mathrm{a}$ & 51.49 a & $\begin{array}{l}31.73 \\
a\end{array}$ & 38.73 \\
\hline 32nd & Dominga & $1.87 \mathrm{~d}$ & $\begin{array}{l}3.45 \\
f\end{array}$ & $e^{7.82}$ & $\begin{array}{l}7.70 \\
d\end{array}$ & $\begin{array}{l}1.37 \\
b\end{array}$ & $5.64 \mathrm{~d}$ & $\begin{array}{l}1.70 \\
c\end{array}$ & $41.12 \mathrm{a}$ & $48.51 \mathrm{~b}$ & $\begin{array}{l}29.03 \\
a\end{array}$ & 36.81 \\
\hline $33 r d$ & $\begin{array}{l}\text { PROG } \\
135\end{array}$ & $1.98 \mathrm{~d}$ & $\begin{array}{l}4.53 \\
e\end{array}$ & $\begin{array}{l}13.57 \\
c\end{array}$ & $\begin{array}{l}8.00 \\
d\end{array}$ & $\begin{array}{l}1.97 \\
a\end{array}$ & $4.06 \mathrm{~d}$ & $\begin{array}{l}2.37 \\
b\end{array}$ & 39.78 a & $41.67 \mathrm{c}$ & $\begin{array}{l}27.09 \\
b\end{array}$ & 30.72 \\
\hline 34th & $\begin{array}{l}\text { PROG } \\
142\end{array}$ & $1.82 \mathrm{~d}$ & $e^{4.51}$ & $e^{7.58}$ & $\begin{array}{l}7.77 \\
d\end{array}$ & $\begin{array}{l}1.44 \\
b\end{array}$ & $5.39 \mathrm{~d}$ & $\begin{array}{l}2.80 \\
a\end{array}$ & $40.64 \mathrm{a}$ & $48.02 \mathrm{~b}$ & $\begin{array}{l}31.20 \\
a\end{array}$ & 27.64 \\
\hline 35th & Camta & $1.51 \mathrm{e}$ & $\begin{array}{l}1.81 \\
\mathrm{~g}\end{array}$ & $e^{5.02}$ & $\begin{array}{l}10.27 \\
c\end{array}$ & $\begin{array}{l}1.85 \\
a\end{array}$ & $5.55 \mathrm{~d}$ & $\begin{array}{l}1.89 \\
\mathrm{C}\end{array}$ & $41.28 \mathrm{a}$ & $49.99 \mathrm{a}$ & $\begin{array}{l}31.69 \\
a\end{array}$ & 21.94 \\
\hline CV (\%) & & 5.36 & 10.38 & 13.02 & 7.55 & 9.72 & 10.44 & 12.19 & 6.45 & 7.71 & 10.35 & - \\
\hline
\end{tabular}


Table 4

Physicochemical attributes of red-ripe fruit and ranks of acerola genotypes based on the multivariate selection index used to identify the most promising genotypes for fresh fruit consumption. All genotypes were harvested in the 2020 growing season, in the São

Francisco Valley, Petrolina, PE, Brazil.

\begin{tabular}{|c|c|c|c|c|c|c|c|c|c|c|c|c|}
\hline & Genotype & $\begin{array}{l}\text { Diameter } \\
\text { (cm) }\end{array}$ & $\begin{array}{l}\text { Mass } \\
(\mathrm{g})\end{array}$ & $\begin{array}{l}\mathrm{FF} \\
(\mathrm{N})\end{array}$ & $\begin{array}{l}\text { SS } \\
(\%)\end{array}$ & $\begin{array}{l}\text { TA } \\
(\%)\end{array}$ & SS/TA & $\begin{array}{l}\text { AA } \\
(\%)\end{array}$ & Lightness & Chroma & $\begin{array}{l}\text { Hue } \\
\text { angle }\end{array}$ & Ranking \\
\hline $1 \mathrm{st}$ & $\begin{array}{l}\text { BRS } \\
\text { Rubra }\end{array}$ & $2.35 b^{*}$ & $\begin{array}{l}6.38 \\
b\end{array}$ & $\begin{array}{l}14.31 \\
b\end{array}$ & $\begin{array}{l}12.40 \\
b\end{array}$ & $\begin{array}{l}0.79 \\
\mathrm{e}\end{array}$ & $\begin{array}{l}15.84 \\
a\end{array}$ & $\begin{array}{l}0.93 \\
d\end{array}$ & $45.03 \mathrm{~b}$ & $48.28 \mathrm{a}$ & $\begin{array}{l}35.70 \\
b\end{array}$ & 99.54 \\
\hline 2nd & $\begin{array}{l}\text { PROG } \\
052\end{array}$ & $2.39 \mathrm{a}$ & $\begin{array}{l}7.34 \\
a\end{array}$ & $\begin{array}{l}12.94 \\
b\end{array}$ & $\begin{array}{l}12.17 \\
b\end{array}$ & $\begin{array}{l}1.38 \\
c\end{array}$ & $8.87 c$ & $\begin{array}{l}1.00 \\
d\end{array}$ & $36.82 \mathrm{c}$ & $41.08 \mathrm{c}$ & $\begin{array}{l}28.18 \\
d\end{array}$ & 78.96 \\
\hline $3 r d$ & $\begin{array}{l}\text { Costa } \\
\text { Rica }\end{array}$ & $2.44 \mathrm{a}$ & $\begin{array}{l}7.65 \\
a\end{array}$ & $\begin{array}{l}9.70 \\
c\end{array}$ & $\begin{array}{l}12.00 \\
b\end{array}$ & $\begin{array}{l}1.39 \\
\mathrm{C}\end{array}$ & $8.71 \mathrm{c}$ & $\begin{array}{l}1.16 \\
d\end{array}$ & $39.98 \mathrm{~b}$ & $47.73 \mathrm{~b}$ & $\begin{array}{l}30.38 \\
\mathrm{c}\end{array}$ & 73.81 \\
\hline 4th & $\begin{array}{l}\text { PROG } \\
069\end{array}$ & $2.22 \mathrm{~b}$ & $\begin{array}{l}6.18 \\
b\end{array}$ & $\begin{array}{l}13.96 \\
b\end{array}$ & $\begin{array}{l}10.10 \\
d\end{array}$ & $\begin{array}{l}0.98 \\
\mathrm{e}\end{array}$ & $\begin{array}{l}10.40 \\
b\end{array}$ & $\begin{array}{l}1.50 \\
\mathrm{C}\end{array}$ & $33.14 \mathrm{~d}$ & $38.47 \mathrm{c}$ & $\begin{array}{l}25.76 \\
d\end{array}$ & 72.45 \\
\hline 5th & Olivier & $2.36 \mathrm{a}$ & $\begin{array}{l}5.83 \\
b\end{array}$ & $\begin{array}{l}9.34 \\
\mathrm{c}\end{array}$ & $\begin{array}{l}12.10 \\
b\end{array}$ & $\begin{array}{l}1.16 \\
d\end{array}$ & $\begin{array}{l}10.47 \\
b\end{array}$ & $\begin{array}{l}1.20 \\
d\end{array}$ & $36.26 \mathrm{c}$ & $35.50 \mathrm{~d}$ & $\begin{array}{l}29.77 \\
c\end{array}$ & 72.04 \\
\hline 6th & $\begin{array}{l}\text { PROG } \\
215\end{array}$ & $2.29 \mathrm{~b}$ & $\begin{array}{l}5.81 \\
b\end{array}$ & $\begin{array}{l}17.55 \\
a\end{array}$ & $\begin{array}{l}9.67 \\
d\end{array}$ & $\begin{array}{l}1.29 \\
\mathrm{c}\end{array}$ & $7.62 \mathrm{c}$ & $\begin{array}{l}1.18 \\
d\end{array}$ & $40.35 \mathrm{~b}$ & $49.67 \mathrm{a}$ & $\begin{array}{l}34.98 \\
b\end{array}$ & 71.35 \\
\hline 7th & Cabocla & $2.40 \mathrm{a}$ & $\begin{array}{l}7.20 \\
a\end{array}$ & $\begin{array}{l}14.98 \\
b\end{array}$ & $\begin{array}{l}10.90 \\
c\end{array}$ & $\begin{array}{l}1.41 \\
\mathrm{C}\end{array}$ & $7.84 \mathrm{c}$ & $\begin{array}{l}1.80 \\
b\end{array}$ & $33.23 \mathrm{~d}$ & $34.93 \mathrm{~d}$ & $\begin{array}{l}22.25 \\
\mathrm{e}\end{array}$ & 70.72 \\
\hline 8th & UEL 03 & $1.79 \mathrm{~d}$ & $\begin{array}{l}2.90 \\
d\end{array}$ & $\begin{array}{l}10.46 \\
C\end{array}$ & $\begin{array}{l}13.87 \\
a\end{array}$ & $\begin{array}{l}0.89 \\
\mathrm{e}\end{array}$ & $\begin{array}{l}15.55 \\
a\end{array}$ & $\begin{array}{l}0.94 \\
d\end{array}$ & $34.15 \mathrm{~d}$ & $40.20 \mathrm{c}$ & $\begin{array}{l}20.50 \\
\mathrm{e}\end{array}$ & 69.29 \\
\hline 9th & Okinawa & $2.47 \mathrm{a}$ & $\begin{array}{l}8.00 \\
a\end{array}$ & $\begin{array}{l}18.60 \\
a\end{array}$ & $e^{8.10}$ & $\begin{array}{l}1.65 \\
b\end{array}$ & $4.91 \mathrm{e}$ & $\begin{array}{l}1.46 \\
c\end{array}$ & $43.78 \mathrm{~b}$ & $46.54 \mathrm{~b}$ & $\begin{array}{l}31.32 \\
c\end{array}$ & 68.44 \\
\hline 10th & ACO 17 & $2.10 \mathrm{c}$ & $\begin{array}{l}4.47 \\
\mathrm{C}\end{array}$ & $\begin{array}{l}12.73 \\
b\end{array}$ & $\begin{array}{l}11.50 \\
c\end{array}$ & $\begin{array}{l}1.15 \\
d\end{array}$ & $\begin{array}{l}10.02 \\
b\end{array}$ & $\begin{array}{l}1.30 \\
d\end{array}$ & $34.63 \mathrm{~d}$ & $38.02 \mathrm{c}$ & $e^{23.65}$ & 63.81 \\
\hline 11th & $\begin{array}{l}\text { PROG } \\
188\end{array}$ & $2.41 \mathrm{a}$ & $\begin{array}{l}6.36 \\
b\end{array}$ & $\begin{array}{l}10.28 \\
c\end{array}$ & $\begin{array}{l}11.33 \\
\mathrm{C}\end{array}$ & $\begin{array}{l}1.55 \\
b\end{array}$ & $7.47 \mathrm{c}$ & $\begin{array}{l}1.41 \\
\mathrm{C}\end{array}$ & $32.60 \mathrm{~d}$ & $37.95 \mathrm{c}$ & $\mathrm{e}^{21.89}$ & 62.19 \\
\hline 12th & $\begin{array}{l}\text { PROG } \\
102\end{array}$ & $2.15 b$ & $\begin{array}{l}5.42 \\
b\end{array}$ & $\begin{array}{l}18.75 \\
a\end{array}$ & $\begin{array}{l}7.50 \\
\mathrm{e}\end{array}$ & $\begin{array}{l}1.14 \\
d\end{array}$ & $6.61 \mathrm{~d}$ & $\begin{array}{l}1.46 \\
\mathrm{C}\end{array}$ & $37.71 \mathrm{c}$ & $43.45 \mathrm{~b}$ & $\begin{array}{l}27.61 \\
d\end{array}$ & 61.48 \\
\hline 13th & $\begin{array}{l}\text { PROG } \\
195\end{array}$ & $2.29 \mathrm{~b}$ & $\begin{array}{l}5.92 \\
b\end{array}$ & $\begin{array}{l}13.46 \\
b\end{array}$ & $\begin{array}{l}11.23 \\
c\end{array}$ & $\begin{array}{l}1.51 \\
b\end{array}$ & $7.45 c$ & $\begin{array}{l}1.70 \\
c\end{array}$ & $39.27 \mathrm{c}$ & $41.76 \mathrm{c}$ & $\begin{array}{l}31.03 \\
c\end{array}$ & 61.18 \\
\hline 14th & $\begin{array}{l}\text { PROG } \\
123\end{array}$ & $2.07 \mathrm{c}$ & $\begin{array}{l}4.50 \\
\mathrm{C}\end{array}$ & $\begin{array}{l}11.47 \\
\mathrm{C}\end{array}$ & $\begin{array}{l}12.63 \\
b\end{array}$ & $\begin{array}{l}1.22 \\
d\end{array}$ & $\begin{array}{l}10.36 \\
b\end{array}$ & $\begin{array}{l}1.60 \\
\mathrm{C}\end{array}$ & $35.61 \mathrm{c}$ & $39.95 \mathrm{c}$ & $\begin{array}{l}27.63 \\
d\end{array}$ & 61.09 \\
\hline 15th & $\begin{array}{l}\text { PROG } \\
023\end{array}$ & $2.24 \mathrm{~b}$ & $\begin{array}{l}5.38 \\
b\end{array}$ & $\begin{array}{l}14.51 \\
b\end{array}$ & $\begin{array}{l}8.00 \\
e\end{array}$ & $\begin{array}{l}1.17 \\
d\end{array}$ & $6.83 \mathrm{~d}$ & $\begin{array}{l}1.29 \\
d\end{array}$ & $34.63 \mathrm{~d}$ & $38.02 \mathrm{c}$ & $e^{23.65}$ & 58.88 \\
\hline 16th & $\begin{array}{l}\text { PROG } \\
233\end{array}$ & $2.25 b$ & $\begin{array}{l}5.73 \\
b\end{array}$ & $\begin{array}{l}10.38 \\
c\end{array}$ & $\begin{array}{l}8.73 \\
e\end{array}$ & 1.15 & $7.56 \mathrm{c}$ & $\begin{array}{l}1.05 \\
d\end{array}$ & $41.59 \mathrm{~b}$ & $46.47 \mathrm{~b}$ & $\begin{array}{l}33.92 \\
b\end{array}$ & 58.40 \\
\hline 17th & ACO 35 & $2.12 \mathrm{c}$ & $\begin{array}{l}4.71 \\
\mathrm{C}\end{array}$ & $\begin{array}{l}11.23 \\
c\end{array}$ & $\begin{array}{l}10.13 \\
d\end{array}$ & $\begin{array}{l}0.97 \\
e^{-9}\end{array}$ & $\begin{array}{l}10.41 \\
b\end{array}$ & $\begin{array}{l}1.92 \\
b\end{array}$ & $40.56 \mathrm{~b}$ & $46.70 \mathrm{~b}$ & $\begin{array}{l}26.47 \\
d\end{array}$ & 57.76 \\
\hline 18th & Valéria & $2.26 \mathrm{~b}$ & $\begin{array}{l}5.08 \\
\mathrm{C}\end{array}$ & $\begin{array}{l}11.56 \\
C\end{array}$ & $\begin{array}{l}10.23 \\
d\end{array}$ & $\begin{array}{l}1.33 \\
\mathrm{c}\end{array}$ & $7.84 \mathrm{c}$ & $\begin{array}{l}1.61 \\
c\end{array}$ & $40.31 \mathrm{~b}$ & $49.73 \mathrm{a}$ & $\begin{array}{l}28.21 \\
d\end{array}$ & 56.06 \\
\hline 19th & BV 07 & $2.25 b$ & $\begin{array}{l}5.91 \\
b\end{array}$ & $\begin{array}{l}8.47 \\
d\end{array}$ & $\begin{array}{l}7.77 \\
\mathrm{e}\end{array}$ & $\begin{array}{l}1.11 \\
d\end{array}$ & $6.99 \mathrm{~d}$ & $\begin{array}{l}0.81 \\
d\end{array}$ & $37.31 \mathrm{c}$ & $42.78 \mathrm{~b}$ & $\begin{array}{l}28.27 \\
d\end{array}$ & 54.94 \\
\hline 20th & CARP 01 & $2.09 \mathrm{c}$ & $\begin{array}{l}4.99 \\
C\end{array}$ & $\begin{array}{l}8.39 \\
d\end{array}$ & $\begin{array}{l}11.90 \\
b\end{array}$ & $\begin{array}{l}1.28 \\
\mathrm{C}\end{array}$ & $9.53 \mathrm{~b}$ & $\begin{array}{l}1.64 \\
c\end{array}$ & $43.19 b$ & $50.20 \mathrm{a}$ & $\begin{array}{l}33.43 \\
b\end{array}$ & 53.33 \\
\hline $21 \mathrm{st}$ & ACO 18 & $2.18 \mathrm{~b}$ & $\begin{array}{l}5.25 \\
c\end{array}$ & $\begin{array}{l}11.10 \\
\mathrm{C}\end{array}$ & $\begin{array}{l}7.90 \\
\mathrm{e}\end{array}$ & $\begin{array}{l}1.19 \\
d\end{array}$ & $6.65 d$ & $\begin{array}{l}1.25 \\
d\end{array}$ & $39.14 \mathrm{c}$ & $45.33 \mathrm{~b}$ & $\begin{array}{l}29.37 \\
C\end{array}$ & 50.71 \\
\hline
\end{tabular}

*Averages followed by the same letter in the column are statistically equal according to the Scott-Knott test (5\%). 


\begin{tabular}{|c|c|c|c|c|c|c|c|c|c|c|c|c|}
\hline & Genotype & $\begin{array}{l}\text { Diameter } \\
\text { (cm) }\end{array}$ & $\begin{array}{l}\text { Mass } \\
\text { (g) }\end{array}$ & $\begin{array}{l}\mathrm{FF} \\
(\mathrm{N})\end{array}$ & $\begin{array}{l}\text { SS } \\
(\%)\end{array}$ & $\begin{array}{l}\text { TA } \\
(\%)\end{array}$ & SS/TA & $\begin{array}{l}\text { AA } \\
(\%)\end{array}$ & Lightness & Chroma & $\begin{array}{l}\text { Hue } \\
\text { angle }\end{array}$ & Ranking \\
\hline 22nd & ACO 10 & $1.84 \mathrm{~d}$ & $\begin{array}{l}3.28 \\
d\end{array}$ & $\begin{array}{l}14.56 \\
b\end{array}$ & $\begin{array}{l}12.00 \\
b\end{array}$ & $\begin{array}{l}1.63 \\
b\end{array}$ & $7.35 \mathrm{c}$ & $\begin{array}{l}0.90 \\
\mathrm{~d}\end{array}$ & $35.67 \mathrm{c}$ & $36.77 \mathrm{c}$ & 25.65 & 49.66 \\
\hline $23 r d$ & $\begin{array}{l}\text { PROG } \\
081\end{array}$ & $2.50 \mathrm{a}$ & $\begin{array}{l}7.55 \\
a\end{array}$ & $\begin{array}{l}10.77 \\
\mathrm{C}\end{array}$ & $\begin{array}{l}9.33 \\
d\end{array}$ & $\begin{array}{l}1.69 \\
b\end{array}$ & $5.50 \mathrm{e}$ & $\begin{array}{l}2.54 \\
a\end{array}$ & $33.39 d$ & $31.45 \mathrm{~d}$ & $\begin{array}{l}23.56 \\
\mathrm{e}\end{array}$ & 49.66 \\
\hline 24th & ACO 03 & $1.90 \mathrm{~d}$ & $\begin{array}{l}3.69 \\
d\end{array}$ & $\begin{array}{l}11.54 \\
c\end{array}$ & $\begin{array}{l}13.87 \\
a\end{array}$ & $\begin{array}{l}1.88 \\
a\end{array}$ & $7.41 \mathrm{c}$ & $\begin{array}{l}1.72 \\
\mathrm{c}\end{array}$ & $44.30 \mathrm{~b}$ & $52.13 \mathrm{a}$ & $\begin{array}{l}34.64 \\
b\end{array}$ & 41.80 \\
\hline 25th & $\begin{array}{l}\text { PROG } \\
046\end{array}$ & $2.20 \mathrm{~b}$ & $\begin{array}{l}5.18 \\
\mathrm{c}\end{array}$ & $\begin{array}{l}12.56 \\
b\end{array}$ & $\begin{array}{l}9.37 \\
d\end{array}$ & $\begin{array}{l}1.81 \\
a\end{array}$ & $5.17 \mathrm{e}$ & $\begin{array}{l}1.87 \\
b\end{array}$ & $51.83 \mathrm{a}$ & $38.64 \mathrm{c}$ & $\mathrm{e}^{21.66}$ & 41.03 \\
\hline 26th & Dominga & $2.04 \mathrm{c}$ & $\begin{array}{l}4.02 \\
d\end{array}$ & $\begin{array}{l}7.54 \\
d\end{array}$ & $\begin{array}{l}10.60 \\
c\end{array}$ & $\begin{array}{l}1.38 \\
c\end{array}$ & $7.70 \mathrm{c}$ & $\begin{array}{l}1.93 \\
b\end{array}$ & $45.79 \mathrm{~b}$ & 52.59 a & $\begin{array}{l}36.73 \\
b\end{array}$ & 37.83 \\
\hline 27th & $\begin{array}{l}\text { PROG } \\
122\end{array}$ & $2.08 \mathrm{c}$ & $\begin{array}{l}4.27 \\
\mathrm{c}\end{array}$ & $\begin{array}{l}10.41 \\
\mathrm{C}\end{array}$ & $\begin{array}{l}9.27 \\
d\end{array}$ & $\begin{array}{l}1.66 \\
b\end{array}$ & $5.59 \mathrm{e}$ & $\begin{array}{l}1.52 \\
c\end{array}$ & $32.47 d$ & $27.66 \mathrm{e}$ & $\begin{array}{l}22.07 \\
\mathrm{e}\end{array}$ & 36.90 \\
\hline 28th & ALHA 06 & $2.15 b$ & $\begin{array}{l}5.05 \\
c\end{array}$ & $\begin{array}{l}7.49 \\
d\end{array}$ & $\begin{array}{l}9.23 \\
d\end{array}$ & $\begin{array}{l}1.42 \\
\mathrm{c}\end{array}$ & $6.52 \mathrm{~d}$ & $\begin{array}{l}2.08 \\
b\end{array}$ & $43.55 b$ & $38.85 \mathrm{c}$ & $\begin{array}{l}31.81 \\
\mathrm{C}\end{array}$ & 36.35 \\
\hline 29th & $\begin{array}{l}\text { PROG } \\
135\end{array}$ & $2.07 c$ & $\begin{array}{l}4.58 \\
c\end{array}$ & $\begin{array}{l}10.15 \\
c\end{array}$ & $\begin{array}{l}9.07 \\
d\end{array}$ & $\begin{array}{l}1.79 \\
a\end{array}$ & $5.08 \mathrm{e}$ & $\begin{array}{l}1.28 \\
d\end{array}$ & $33.30 \mathrm{~d}$ & $35.62 \mathrm{~d}$ & $\mathrm{e}^{21.92}$ & 35.79 \\
\hline 30th & RECI 02 & $2.00 \mathrm{c}$ & $\begin{array}{l}3.73 \\
d\end{array}$ & $\begin{array}{l}7.41 \\
d\end{array}$ & $\begin{array}{l}12.23 \\
b\end{array}$ & $\begin{array}{l}1.65 \\
b\end{array}$ & $7.63 \mathrm{c}$ & $\begin{array}{l}2.22 \\
b\end{array}$ & $35.34 \mathrm{c}$ & $39.10 \mathrm{c}$ & $\begin{array}{l}24.38 \\
\mathrm{e}\end{array}$ & 33.42 \\
\hline $31 \mathrm{st}$ & $\begin{array}{l}\text { PROG } \\
216\end{array}$ & $1.89 \mathrm{~d}$ & $\begin{array}{l}3.60 \\
d\end{array}$ & $\begin{array}{l}7.94 \\
d\end{array}$ & $\begin{array}{l}12.93 \\
b\end{array}$ & $\begin{array}{l}1.78 \\
a\end{array}$ & $7.27 \mathrm{c}$ & $\begin{array}{l}1.93 \\
b\end{array}$ & 37.92 c & $45.78 \mathrm{~b}$ & $\begin{array}{l}27.82 \\
d\end{array}$ & 32.54 \\
\hline 32nd & $\begin{array}{l}\text { PROG } \\
142\end{array}$ & $1.96 \mathrm{c}$ & $\begin{array}{l}3.92 \\
d\end{array}$ & $\begin{array}{l}9.48 \\
\mathrm{C}\end{array}$ & $\begin{array}{l}10.63 \\
c\end{array}$ & $\begin{array}{l}1.64 \\
b\end{array}$ & $6.47 d$ & $\begin{array}{l}2.72 \\
a\end{array}$ & $35.69 \mathrm{c}$ & $38.33 \mathrm{c}$ & $\begin{array}{l}25.17 \\
d\end{array}$ & 26.78 \\
\hline $33 r d$ & $\begin{array}{l}\text { PROG } \\
244\end{array}$ & $1.83 \mathrm{~d}$ & $\begin{array}{l}2.99 \\
d\end{array}$ & $\begin{array}{l}6.95 \\
d\end{array}$ & $\begin{array}{l}8.43 \\
\mathrm{e}\end{array}$ & $\begin{array}{l}1.23 \\
d\end{array}$ & $6.91 \mathrm{~d}$ & $\begin{array}{l}1.65 \\
c\end{array}$ & $38.15 \mathrm{c}$ & $44.93 \mathrm{~b}$ & $\begin{array}{l}24.22 \\
\mathrm{e}\end{array}$ & 26.04 \\
\hline 34th & Barbados & $1.81 \mathrm{~d}$ & $\begin{array}{l}2.86 \\
d\end{array}$ & $\begin{array}{l}7.62 \\
d\end{array}$ & $\begin{array}{l}9.63 \\
d\end{array}$ & $\begin{array}{l}1.86 \\
a\end{array}$ & $5.22 \mathrm{e}$ & $\begin{array}{l}1.55 \\
c\end{array}$ & $35.27 \mathrm{c}$ & $37.77 \mathrm{c}$ & $\begin{array}{l}23.68 \\
\mathrm{e}\end{array}$ & 18.09 \\
\hline 35th & Camta & $1.74 \mathrm{~d}$ & $\begin{array}{l}3.39 \\
d\end{array}$ & $\begin{array}{l}7.49 \\
d\end{array}$ & $\begin{array}{l}10.70 \\
c\end{array}$ & $\begin{array}{l}1.89 \\
a\end{array}$ & $5.69 \mathrm{e}$ & $\begin{array}{l}2.53 \\
a\end{array}$ & $49.57 \mathrm{a}$ & $49.25 \mathrm{a}$ & $\begin{array}{l}45.82 \\
a\end{array}$ & 12.87 \\
\hline CV (\%) & & 6.73 & 15.56 & 15.51 & 8.35 & 9.82 & 11.12 & 20.62 & 8.54 & 7.30 & 9.96 & - \\
\hline
\end{tabular}

The growing season had no effect on the average acerola diameter and mass (Table 2). In the first growing season, the highest fruit diameter and mass were observed in the genotype PROG 052, whereas the lowest diameter was observed in the genotype Camta and the lowest mass in the genotypes ACO 35, PROG 102, and Camta (Table 3). In the second growing season, the highest fruit diameter was observed in the genotypes PROG 052, Costa Rica, Oliver, Cabocla, Okinawa, PROG 188, and PROG 081, and lowest diameter was observed in the genotypes UEL 03, ACO 10, PROG 216, PROG 244, Barbados, and Camta (Table 4). In 2020, the highest fruit mass was observed in the genotypes PROG 052, Costa Rica, Cabocla, Okinawa, and PROG 081, whereas the lowest mass was observed in the genotypes UEL 03, ACO 10, ACO 03, Dominga, RECI, PROG 216, PROG 142, PROG 244, Barbados, and Camta (Table 4).

The average flesh firmness was higher in fruit harvested in 2020, compared to fruit harvested in 2019 growing season (Table 2). In 2019, the highest acerola flesh firmness was observed in the genotype PROG 069, whereas the lowest flesh firmness was observed in the genotypes CARP 01, Oliver, PROG 233, Costa Rica, PROG 215, BV 07, ACO 35, ACO 03, Valéria, Barbados, Dominga, PROG 142, and Camta (Table 3). In 2020, the highest flesh firmness was observed in the genotypes PROG 215, Okinawa, and PROG 102, and the lowest was observed in the genotypes BV 07, CARP 01, Dominga, ALHA 06, RECI 02, PROG 216, PROG 244, Barbados, and Camta (Table 4). 
The average SS content was higher in 2020, compared to 2019 (Table 2). In the first growing season, the highest SS content was observed in the genotype ACO 35, and the lowest in the genotypes CARP 01, PROG 123, PROG 215, PROG 081, ACO 18, BV 07, Barbados, PROG 244, and PROG 046 (Table 3). In the second growing season, the highest SS content was observed in the genotype UEL 03, and ACO 03, whereas the lowest SS content was observed in the genotypes Okinawa, PROG 102, PROG 023, PROG 233, BV 07, ACO 18, and PROG 244 (Table 4).

The average acerola TA was not affected by the growing season (Table 2). In 2019, the highest TA was observed in the genotypes ACO 10, Okinawa, ACO 35, ALHA 06, PROG 188, PROG 216, PROG 244, PROG 135, and Camta, whereas the lowest TA was observed in the genotypes BRS Rubra, Oliver, PROG 123, and PROG 215 (Table 3). In 2020, the highest TA was observed in the genotypes ACO 03 , PROG 046, PROG 135, PROG 216, Barbados, and Camta, and the lowest TA was observed in the genotypes BRS Rubra, PROG 069, UEL 03 and ACO 35 (Table 4).

The average SS/TA ratio was higher in acerolas harvested in 2020, compared to acerolas harvested in 2019 (Table 2). In the first growing season, the highest SS/TA ratio was observed in the genotypes BRS Rubra, Oliver, PROG 233, whereas the lowest SS/TA ratio was observed in the genotypes PROG 052, PROG 081, PROG 122, ALHA 06, PROG 188, Valéria, Barbados, PROG 244, PROG 046, Dominga, PROG 135, PROG 142, and Camta (Table 3). In the second growing season, the highest SS/TA ratio was observed in the genotypes BRS Rubra, and UEL 03, whereas the lowest SS/TA ratio was observed in the genotypes Okinawa, PROG 081, PROG 046, PROG 122, PROG 135, Barbados, and Camta (Table 4).

The average AA content in acerola genotypes was not affected by the growing season (Table 2). In 2019, the highest AA content was observed in the genotype PROG 142, and the lowest in the genotypes BRS Rubra, CARP 01, Oliver, PROG 215, and BV 07 (Table 3). In 2020, the highest AA content was observed in the genotypes PROG 081, PROG 142, and Camta, whereas the lowest AA content was observed in the genotypes BRS Rubra, PROG 052, Costa Rica, Oliver, PROG 215, UEL 03, ACO 17, PROG 023, PROG 233, BV 07, ACO 18, ACO 10, and PROG 135 (Table 4).

The average acerola skin lightness ( $L$ ) was not affected by the growing season (Table 2). In 2029, the highest skin $L$ was observed in the genotypes BRS Rubra, Cabocla, CARP 01, PROG 233, UEL 03, PROG 123, Costa Rica, PROG 215, ACO 18, ACO 10, RECI 02, PROG 195, Okinawa, BV 07, ACO 35, ALHA 06, ACO 03, Barbados, PROG 244, PROG 046, Dominga, PROG 135, PROG 142, and Camta, whereas all other genotypes showed the lowest skin $L$ value (Table 3). In 2020, the highest skin $L$ was observed in the genotypes PROG 046, and Camta, whereas the lowest skin L was observed in the genotypes PROG 069, Cabocla, UEL 03, ACO 17, PROG 188, PROG 023, PROG 081, PROG 122, and PROG 135 (Table 4).

The average acerola skin chroma (C) was higher in fruit harvested in 2019, compared to fruit harvested in 2020 (Table 2). In the first growing season, the highest skin C was observed in the genotypes Cabocla, Costa Rica, BV 07, ACO 03, PROG 244, PROG 046, and Camta, whereas the lowest skin C was observed in the genotypes PROG 069, PROG 023, PROG 122, ALHA 06, and PROG 188 (Table 3). In the second growing season, the highest skin C was observed in the genotypes BRS Rubra, PROG 215, Valéria, CARP 01, ACO 03, Dominga, and Camta, whereas the lowest skin C was observed in the genotypes Oliver, Cabocla, PROG 081, and PROG 135 (Table 4).

The average acerola skin hue angle $\left({ }^{\circ} \mathrm{h}\right)$ was not affected the growing season (Table 2). In 2019, the highest skin ${ }^{\circ} \mathrm{h}$ was observed in the genotypes PROG 052, BRS Rubra, Cabocla, CARP 01, PROG 233, UEL 03, PROG 123, Costa Rica, PROG 215, ACO 18, ACO 10, RECI 02, PROG 195, Okinawa, BV 07, ALHA 06, ACO 03, PROG 244, PROG 046, Dominga, PROG 142, and Camta, whereas the lowest skin oh was observed in the genotypes PROG 069, PROG 023, PROG 122, Valéria, and PROG 102 (Table 3). In 2020, the highest skin ${ }^{\circ} \mathrm{h}$ was observed in the genotype Camta, whereas the lowest skin ${ }^{\circ} \mathrm{h}$ was observed in the genotypes Cabocla, UEL 03, ACO 17, PROG 188, PROG 023, PROG 081, PROG 046, PROG 122, PROG 135, RECI 02, PROG 244, and Barbados (Table 4).

According to the selection index, in the first growing season the best genotype for fresh fruit consumption was PROG 052 (Table 3), which stood out mainly for its combined values of high diameter $\left(2.79 \mathrm{~cm}_{\text {fruit }}{ }^{-1}\right)$, mass $\left(10.80 \mathrm{~g} \mathrm{fruit}^{-1}\right)$ and flesh firmness (17.38 $\mathrm{N}$ ), as demonstrated by the PCA (Fig. 2). In the second growing season, the genotype PROG 052 was also between the best genotypes for fresh consumption, being classified with the second highest selection index (Table 4).

The genotype BRS Rubra had the second and first selection indexes in 2019 and 2020, due to its high SS contents of $9.33 \%$ and $12.40 \%$, low TA of $0.93 \%$ and $0.79 \%$, and high SS/TA ratios of 10.08 and 15.84 , respectively (Tables 3 and 4 ).

Page $10 / 18$ 
The acerola genotypes Cabocla and Costa Rica had the third highest selection index in the first and second growing seasons, respectively (Tables 3 and 4). The genotype Cabocla had a high SS/TA ratio of 8.78 in 2019, as pointed out by the PCA (Fig. 2). In the second growing season, the high selection index of the genotype Costa Rica is mainly attributed to its high diameter, mass and SS content (Tables 3 and 4).

The genotype PROG 069 had the fourth highest selection index in both growing seasons (Tables 3 and 4). In 2019, the genotype PROG 069 had the highest flesh firmness among all genotypes, while in 2020 it stood out for its high flesh firmness and SS/TA ratio, as well as low TA (Tables 3 and 4).

According to the principal component analysis (PCA), the data set of the first growing season was represented in the first three principal components, which had eigenvalues higher than 1 and together represented $84.5 \%$ of the total data variability (Fig. 2). PC1 explained $34.05 \%$ of the variance in the dataset, whereas PC2 was responsible for $33.42 \%$ and PC3 for $17.06 \%$. The main variables correlated with the first principal component were ascorbic acid and titratable acidity that had PC1 $>0$, and diameter and mass that had PC1 < 0 (Fig. 2). The first genotypes raked by the multivariate selection index, PROG 052, BRS Rubra, Cabocla and PROG 069 had PC1 < 0 (Fig. 2). The second principal component was positively correlated with SS/TA ratio and negatively correlated with flesh firmness (Fig. 2). Having high flesh firmness, the genotypes PROG 052 and PROG 069 were negatively correlated with PC2, while the genotype BRS Rubra had a high positive correlation with PC2 due to the high SS/TA ratio (Fig. 2). Soluble solids content was better explained by the third principal component, in a positive axis, where the first four ranked genotypes were also located.

The second growing season was significantly represented in the first two principal components, which explained $68.4 \%$ of the data variability (Fig. 3). PC1 explained $39.26 \%$ of the data variance and PC2 was responsible for $29.18 \%$. The physicochemical attributes correlated with the PC1 were ascorbic acid and titratable acidity $(P C 1>0)$, as well as diameter, mass and flesh firmness $(P C 1<0)($ Fig. 3). The genotypes BRS Rubra, PROG 052, Costa Rica and PROG 069 ranked in the first places in the second growing season had PC1 $<0$ (Fig. 3). In the PC2, the major contribution variables were SS and SS/TA ratio, both with positive values (Fig. 3). The first four ranked genotypes in the second growing season showed PC2 >0 (Fig. 3).

\section{Discussion}

The average diameter and mass of acerola fruit were higher than those genotypes commonly cultivated in the São Francisco Valley, such as 'Flor Branca', 'Sertaneja', 'Okinawa' and 'Costa Rica' (Batista et al., 2015). Fruit diameter and mass are important traits for genotype selection due to the fact that larger fruit can yield greater amounts of pulp, which is more desirable for fresh consumption (Carpentieri-Pípolo et al., 2000). Fresh market requirements have stablished that acerola destinated for fresh consumption must have a minimum diameter of $1.5 \mathrm{~cm}$ and mass of $4 \mathrm{~g}$ (Brazilian Fruit Institute, 1995). In that case, all genotypes evaluated in our study presented average diameter values above the minimum in both growing seasons, while the minimum fruit mass was observed in 27 (77.1\%) and $25(71.4 \%)$ genotypes in 2019 and 2020, respectively.

Acerola is a very fragile and perishable fruit mainly due to the accelerated loss of flesh firmness during ripening (Carrington and King, 2002; Quoc et al., 2015; Souza et al., 2017), which has been reported to be associated with increasing activity of cell wall degrading enzymes and loss of cellular turgor pressure (Santos et al., 2019). Indeed, ripe acerolas have lower flesh firmness, compared to other fruit species (Batista et al., 2015), making the fruit more susceptible to mechanical damage and losses. In that case, selection of genotypes with higher flesh firmness is important to guarantee longer postharvest life and lower losses. In our study, red-ripe acerolas had average flesh firmness of $10.11 \mathrm{~N}$ in 2019 and $11.32 \mathrm{~N}$ in 2020, which were higher than the flesh firmness range of 2.56-6.48 N, observed in 45 acerola genotypes cultivated in another Semi-arid region in Brazil (Moura et al., 2007). These results suggest that the genotypes evaluated in our study have high potential for selecting the ones more resistant to mechanical damage and with longer postharvest life, which can be used for commercial cultivation, as well as in breeding programs to obtain new cultivars with better quality traits for the fresh market.

In general, acerola is a very acidic fruit (Delva and Schneider, 2013). In that case, breeding programs for fresh fruit consumption must select fruit with high SS content and low TA in order to improve acerola consumer quality (Matsuura et al., 2001). In our study, red-ripe fruit had average SS content of $8.82 \%$ and $10.50 \%$, and SS ranges of $6.03-13.07 \%$ and $7.50-13.87 \%$ in the first and second growing seasons, respectively. Other studies have also observed a wide variation in acerola SS content, ranging from 3.33-11.75\%, under Semi-arid conditions (Moura et al., 2007; Ribeiro and Freitas, 2020). Although fruit quality is mostly genetically controlled, environmental conditions such as solar radiation and irrigation can also influence acerola SS content. Alves et al. (1999) reported that 
excessive irrigation may cause a dilution effect of fruit SS content. Therefore, adequate crop management practices can enhance sugar accumulation in the fruit, guaranteeing high quality of acerolas intended for fresh consumption that have high sugar contents such as the ACO 35 in the first growing season and UEL 03 and ACO 03 in the second growing season.

TA values observed in our study were slightly higher than those found by Moura et al. (2007) in 45 acerola genotypes $(0.53-1.52 \%$ of malic acid).

Although the SS and TA values have an important effect on consumer quality, the SS/TA ratio has an even more important role on determining fruit quality because it takes into account the balance between the sweet/acid taste of the fruit. Therefore, the SS/TA ratio is more important than each attribute separately, since these isolated parameters may represent a false indicative of fruit flavor (Cavalcante et al., 2007). Ribeiro and Freitas (2020) found average SS/TA values of 5.06 and 4.57 in 'Flor Branca' and 'Junko' acerolas, which are among the main acerola cultivars grown in Brazil. In our study, a wider SS/TA ratio range was observed among genotypes, which makes it possible to select the genotypes with higher ratios, such as BRS Rubra, Oliver, PROG 233 in the first growing season and BRS Rubra, and UEL 03 in the second growing season, for fresh fruit consumption, as well as for breeding programs aiming to develop new acerola genotypes with higher consumer quality.

Acerolas have a reduction in AA content during ripening due to the increasing activity of the ascorbate oxidase, which is the main enzyme responsible for the degradation of ascorbic acid during acerola ripening (Butt, 1980). Other studies have also associated the degradation of ascorbic acid with biochemical oxidation, after detecting the presence of 3-hydroxy-2-pyone, an oxidative breakdown product of ascorbic acid, in ripe acerolas (Vendramini and Trugo, 2000). The red-ripe acerola AA content has been reported to be determined by genetic factors and environmental factors such as orchard sunlight, temperature, relative humidity and water availability, as well as postharvest handling and storage conditions (Delva and Schneider, 2013; Matsuura et al., 2001). In addition, fruit from seed propagated plants usually have lower AA content than those from asexually propagated plants (Mohammed, 2011).

All over the world, medicinal use of acerola fruit is very common against flus and colds, pulmonary disturbance, liver ailments and irregularities of the gall bladder (Assis et al., 2001). Ascorbic acid is the main bioactive compound found in acerolas, due to its high antioxidant activity, besides its key role on the biosynthesis of collagen, carnitine and neurotransmitters (Prakash and Baskaran, 2018). Siqueira et al. (2020) found that vitamin $C$ is the cheapest nutrient in the Brazilian diet, at a cost of 1 cent for $30 \%$ of the daily recommendations of this nutrient, when consumed in the form of acerola juice. In addition, acerolas have also been used in the pharmaceutical and food industries for the production of medicines or enrichment of industrialized foods (Benjamin et al., 2015). Although high AA content is desirable for the industry, our results and previous studies have shown a high and positive correlation between acerola AA content and TA, which can affect SS/TA ratio and fruit flavor (Matsuura et al., 2001; Cavalcante et al., 2007; Moura et al., 2007; Magalhães et al., 2018; Maranhão Ribeiro et al., 2018). Therefore, acerola intended for fresh consumption must have enough AA content to reach the low daily recommendations that will also guarantee a high SS/TA ratio required for good fruit flavor (Cavalcante et al., 2007).

Ripening related color changes occur due to chlorophyll degradation, conversion of chloroplasts to chromoplasts and synthesis of carotenoids and anthocyanins in acerola fruit (Alves et al., 1995; Rosso and Mercadante, 2005). Considering that all genotypes in our study were cultivated in the same environment, the observed variation on skin hue angle could be attributed to different genetic control of chlorophyll degradation and synthesis of carotenoids and anthocyanins in the fruit (Lima et al., 2005; Ribeiro and Freitas, 2020), resulting in genotypes with more intense yellow/red color than others. In general, the observed hue angle values were close to that found in 'Junko' acerolas (red color, 24.9 ${ }^{\circ}$ ) and lower than that found in 'Flor Branca' acerolas (yellow color, 60.8 ${ }^{\circ}$ ), evaluated by Ribeiro and Freitas (2020). The red color development takes place at the final stages of ripening due to increasing synthesis of anthocyanins in the skin tissue, which makes skin color an important quality trait to determine acerola harvest maturity (Delva and Schneider, 2013). In addition to their impact on the visual attraction to consumers, anthocyanins are also important in acerolas for their health properties, since these pigments have high antioxidant, anti-inflammatory, antihypertensive, anti-atherosclerotic and anticancer activities, reducing oxidative damage caused by free radicals to the human organism (Alvarez-Suarez et al., 2017; Souza et al., 2014).

During ripening, acerola skin lightness decreases due to changes from light green to dark red color (Ribeiro and Freitas, 2020). The average lightness value observed for all acerola genotypes in this study is higher than those found in the acerola cultivars Okinawa, Sertaneja, Flor Branca and Costa Rica (20.48 to 23.68) (Batista et al., 2015). The same authors also found a lower hue angle in these genotypes, which indicates that purple-colored acerola cultivars are darker. Chroma represents saturation or purity of color, indicating 
dull colors (low chroma) or vivid color (high chroma). The skin chroma values observed in our study suggest that all genotypes had a vivid color due to the high chroma. In addition, fruit produced in the first growing season had higher chroma (more vivid color) than fruit produced in the second growing season.

In our study, the selection index was applied to rank and identify red-ripe acerola genotypes with the highest potential for fresh consumption. The multivariate selection index combines different quality trait variables (diameter, mass, flesh firmness, SS, TA, SS/TA, AA) of acerola fruit into a single index that is used to rank genotypes based on their quality for fresh consumption (Barth et al., 2020; Poggetti et al., 2017). The best genotype for fresh fruit consumption in the first production cycle was PROG 052, which stood out mainly due to its combined values of high diameter, mass and flesh firmness. These have been suggested to be the most important physical parameters to be considered when choosing acerola genotypes for fresh fruit consumption (Semensato and Pereira, 2000). The genotype PROG 052 also had the second highest selection index in the second growing season, showing that environmental changes had limited effect on fruit quality changes, maintaining this genotype among the best ones for fresh consumption.

According to the selection index, the genotype BRS Rubra ranked second and first in 2019 and 2020, respectively, due to its combination of high SS content and SS/TA ratio, as well as low TA and AA values. These findings corroborate with previously studies accomplished by Godoy et al. (2008) and Mamede et al. (2009). In these studies, comparations among acerola genotypes have shown that BRS Rubra had the highest SS content, as well as the lowest TA and the best consumer acceptance.

The genotypes Cabocla and Costa Rica had the third highest selection index in 2019 and 2020, respectively. Cabocla is a Brazilian variety released in 2002 for fresh fruit consumption, as well as pulp and juice production (Ritzinger and Ritzinger, 2011; Ritzinger et al., 2017). According to our results and previous studies, this genotype has high fruit SS/TA ratio, making it suitable for the fresh market (Godoy et al., 2008). The genotype Costa Rica had a high selection index due to high fruit diameter, mass and SS content, which was also observed in the study accomplished by Batista et al. (2015).

The genotype PROG 069 ranked fourth in both growing seasons, according to the selection index. One of the most important quality traits observed in the genotype PROG 069 was the high fruit flesh firmness, which increases resistance to mechanical damage and postharvest live of the fruit (Quoc et al., 2015; Souza et al., 2017).

\section{Conclusions}

A high variability of physicochemical quality was observed among the acerola genotypes produced under semi-arid conditions.

The multivariate selection index was a powerful tool for identifying genotypes with high potential for fresh consumption, since it allowed selecting genotypes with multiple desirable traits.

The acerola genotypes PROG 052, BRS Rubra, Cabocla, Costa Rica and PROG 069 showed the highest potential for fresh consumption due to the presence of multiple desirable traits such as high diameter, mass, flesh firmness, SS, and SS/AT ratio, as well as lower acidity.

\section{Declarations}

\subsection{Funding}

The authors would like to thank for the financial support from Embrapa, as well as for the scholarships funded by the Coordenação de Aperfeiçoamento de Pessoal de Nível Superior (CAPES), Conselho Nacional de Desenvolvimento Científico e Tecnológico (CNPq), and Fundação de Amparo a Ciência e Tecnologia do Estado de Pernambuco (FACEPE).

\subsection{Conflicts of interest/Competing interests}

The authors declare that they have not conflict of interests/competing interests.

\subsection{Availability of data and material}

The data are available. 


\subsection{Authors' contributions}

Maria Aparecida Rodrigues Ferreira: fruit harvest, physicochemical and statistical analyzes and manuscript writing; João Claudio Vilvert: statistical analysis and manuscript writing; Bárbara Orrana Sobreira da Silva and lanca Carneiro Ferreira: fruit harvest and physicochemical analyzes; Flávio de França Souza: funding, orchard management and manuscript writing; Sérgio Tonetto de Freitas: funding, conceptualization, methodology, manuscript writing and submission.

\section{References}

1. Alvarez-Suarez JM, Giampieri F, Gasparrini M, Mazzoni L, Santos-Buelga C, González-Paramás AM, Forbes-Hernández TY, Afrin S, Páez-Watson T, Quiles JL, Battino M (2017) The protective effect of acerola (Malpighia emarginata) against oxidative damage in human dermal fibroblasts through the improvement of antioxidant enzyme activity and mitochondrial functionality. Food Funct 8:3250-3258. https://doi.org/10.1039/c7fo00859g

2. Alves RE, Chitarra AB, Chitarra MIF (1995) Postharvest physiology of acerola (Malpighia emarginata D.C.) fruits: Maturation changes, respiratory activity and refrigerated storage at ambient and modified atmospheres. Acta Hortic 370:223-229. https://doi.org/10.17660/ActaHortic.1995.370.35

3. Alves RE, Filgueiras HAC, Mosca JL, Menezes B (1999) Brazilian experience on the handling of acerola fruits for international trade: Harvest and postharvest recommendations. Acta Hortic 485:31-36. https://doi.org/10.17660/ActaHortic.1999.485.3

4. Assis SA, Fernandes FP, Martins ABG, Oliveira OMMF (2008) Acerola: importance, culture conditions, production and biochemical aspects. Fruits 63:93-101. https://doi.org/10.1051/fruits:2007051

5. Assis SA, Lima DC, Oliveira OMMF (2001) Activity of pectinmethylesterase, pectin content and vitamin C in acerola fruit at various stages of fruit development. Food Chem 74:133-137. https://doi.org/10.1016/S0308-8146(01)00104-2

6. Barth E, Resende JTV, Moreira AFP, Mariguele KH, Zeist AR, Silva MB, Stulzer GCG, Mafra JGM, Gonçalves LSA, Roberto SR, Youssef K (2020) Selection of experimental hybrids of strawberry using multivariate analysis. Agronomy 10:598. https://doi.org/10.3390/agronomy 10040598

7. Batista PF, Lima MAC, Trindade DCG, Alve R.E (2015) Quality of different tropical fruit cultivars produced in the lower basin of the São Francisco Valley. Rev Ciênc Agron 46:176-184. https://doi.org/10.1590/S1806-66902015000100021

8. Benjamin SR, Oliveira Neto JR, Macedo IYL, Bara MTF, Cunha LC, Carvalho LAF, Gil ES (2015) Electroanalysis for quality control of acerola (Malpighia emarginata) fruits and their commercial products. Food Anal Methods 8:86-92. https://doi.org/10.1007/s12161-014-9872-0

9. Bertini CHCDM, Almeida WSD, Silva APMD, Silva JWL, Teófilo EM (2010) Análise multivariada e índice de seleção na identificação de genótipos superiores de feijão-caupi. Acta Scientiarum Agronomy 32:613-619.

http://dx.doi.org/10.4025/actasciagron.v32i4.4631

10. Brazilian Fruit Institute (1995) Soluções fruta a fruta: acerola. IBRAF, São Paulo.

11. Butt VS (1980) Direct oxidases and related enzymes, in: Stumpf PK, Conn EE (Eds.). The Biochemistry of Plants: A Comprehensive Treatise pp. 81-123.

12. Carpentieri-Pípolo V, Destro D, Prete CEC, Gonzales MGN, Popper I, Zanatta S, Da Silva FAM (2000) Seleção de genótipos parentais de acerola com base na divergência genética multivariada. Pesq Agropec Bras 35:1613-1619. https://doi.org/10.1590/s0100-204×2000000800014

13. Carrington CMS, King RAG (2002) Fruit development and ripening in Barbados cherry, Malpighia emarginata DC. Sci Hortic 92:17. https://doi.org/10.1016/S0304-4238(01)00268-0

14. Cavalcante ÍHL, Beckmann MZ, Martins ABG, Campos MCC (2007) Preliminary selection of acerola genotypes in Brazil. Fruits 62:27-34. https://doi.org/10.1051/fruits:2006046

15. Delva L, Schneider RG (2013) Acerola (Malpighia emarginata DC): Production, postharvest handling, nutrition, and biological activity. Food Rev Int 29:107-126. https://doi.org/10.1080/87559129.2012.714433

16. Godoy RCB, Matos ELS, Amorim TS, Souza Neto MA, Ritzinger R, Waszczynskyj N (2008) Avaliação de genótipos e variedades de acerola para consumo in natura e para elaboração de doces. Bol Cent Pesqui Process Aliment 26:197-204.

https://doi.org/10.5380/cep.v26i2.13274

Page $14 / 18$ 
17. Hanamura T, Uchida E, Aoki H (2008) Changes of the composition in acerola (Malpighia emarginata DC.) fruit in relation to cultivar, growing region and maturity. J Sci Food Agric 88:1813-1820. https://doi.org/10.1002/jsfa

18. Hoehn E, Gasser F, Naepflin B, Ladner J (2005) Consumer expectations and soluble solids, acidity and firmness of plums (Prunus domestica Cacaks Beauty). Acta Hortic 682:665-672 https://doi.org/10.17660/ActaHortic.2005.682.86

19. Lima VLAG, Mélo EA, Maciel MIS, Prazeres FG, Musser RS, Lima DES (2005) Total phenolic and carotenoid contents in acerola genotypes harvested at three ripening stages. Food Chem 90:565-568. https://doi.org/10.1016/j.foodchem.2004.04.014

20. Magalhães DS, Rufni JCM, Alburquerque AS, Viol RE, Fagundes MCP, Menezes TP (2018) Genetic diversity among accessions of acerola based on the quality of fruits. Comun Sci 9:133-141. https://doi.org/10.14295/CS.v9i2.2961

21. Mamede MEO, Miranda MPS, Ritzinger R, Godoy RCB, Velozo ES (2009) Physico-chemical and sensorial evaluation of new varieties of acerola. Br Food J 111:387-395. https://doi.org/10.1108/00070700910951867

22. Maranhão Ribeiro CMC, Sousa TPA, Holschuh HJ, Ribeiro MTJB, Silva SM, Maciel MIS (2018) Fruit development and ripening of acerola 'Okinawa' cultivar. Acta Hortic 1198:199-204. https://doi.org/10.17660/ActaHortic.2018.1198.32

23. Matsuura FCAU, Cardoso RL, Folegatti MIS, Oliveira JRP, Oliveira JAB, Santos DB (2001) Avaliações físico-químicas em frutos de diferentes genótipos de acerola (Malpighia punicifolia L.). Rev Bras Frutic 23:602-606. https://doi.org/10.1590/s010029452001000300032

24. Mezadri T, Fernández-Pachón MS, Villaño D, García-Parrilla MC, Troncoso AM (2006) El fruto de la acerola: composición y posibles usos alimenticios. Arch Latinoam Nutr 56:101-109.

25. Mohammed M (2011) Acerola (Malpighia emarginata DC.), in: Yahia EM (Ed.). Postharvest Biology and Technology of Tropical and Subtropical Fruits: Açai to Citrus. Woodhead Publishing Limited, Cambridge, pp. 27-47.

26. Moura CFH, Alves RE, Figueiredo RW, Paiva JR (2007) Avaliações físicas e físico-químicas de frutos de clones de aceroleira (Malpighia emarginata D.C.). Rev Ciênc Agron 38:52-57.

27. Mulamba NN, Mock JJ (1978) Improvement of yield potential of the Eto Blanco maize (Zea mays L.) population by breeding for plant traits. Egypt J Genet Cytol 7:40-51.

28. Poggetti L, Ermacora P, Cipriani G, Pavan F, Testolin R (2017) Morphological and carpological variability of walnut germplasm (Juglans regia L.) collected in North-Eastern Italy and selection of superior genotypes. Sci Hortic 225:615-619. https://doi.org/10.1016/j.scienta.2017.07.056

29. Prakash A, Baskaran R (2018) Acerola, an untapped functional superfruit: a review on latest frontiers. J Food Sci Technol 55:3373-3384. https://doi.org/10.1007/s13197-018-3309-5

30. Quoc LPT, Hoa DP, Ngoc HTB, Phi TTY (2015) Effect of xanthan gum solution on the preservation of acerola (Malpighia glabra L.). Cercet Agron Mold 48:89-97. https://doi.org/10.1515/cerce-2015-0045

31. Ribeiro BS, Freitas ST (2020) Maturity stage at harvest and storage temperature to maintain postharvest quality of acerola fruit. Sci Hortic 260:108901. https://doi.org/10.1016/j.scienta.2019.108901

32. Ritzinger R, Kobayashi AK, Oliveira JRP (2003) A cultura da aceroleira. Embrapa Mandioca e Fruticultura, Cruz das Almas.

33. Ritzinger R, Ritzinger CHSP (2011) Acerola. Inf Agropec 32:17-25.

34. Ritzinger R, Ritzinger CHSP, Fonseca N, Machado CF (2017) Advances in the propagation of acerola. Rev Bras Frutic 40:e928. https://doi.org/10.1590/0100-29452018928

35. Rosso VV, Mercadante AZ (2005) Carotenoid composition of two Brazilian genotypes of acerola (Malpighia punicifolia L.) from two harvests. Food Res Int 38:1073-1077. https://doi.org/10.1016/j.foodres.2005.02.023

36. Santos CP, Batista MC, Saraiva KDC, Roque ALM, Miranda RS, Silva LMA, Moura CFH, Alves Filho EG, Canuto KM, Costa JH (2019) Transcriptome analysis of acerola fruit ripening: insights into ascorbate, ethylene, respiration, and softening metabolisms. Plant Mol Biol 101:269-296. https://doi.org/10.1007/s11103-019-00903-0

37. Semensato LR, Pereira AS (2000) Características de frutos de genótipos de aceroleira cultivados sob elevada altitude. Pesq Agropec Bras 35:2529-2536. https://doi.org/10.1590/s0100-204x2000001200024

38. Siqueira KB, Binoti ML, Nunes RM, Borges CAV, Pilati AF, Marcelino GW, Gama MAS, Silva PHF (2020) Custo benefício dos nutrientes dos alimentos consumidos no Brasil. Ciênc Saúde Coletiva 25:1129-1135. https://doi.org/10.1590/1413-

81232020253.11972018

Page 15/18 
39. Souza KO, Moura CFH, Brito ES, Miranda MRA (2014) Antioxidant compounds and total antioxidant activity in fruits of acerola from cv. Flor Branca, Florida Sweet and BRS 366. Rev Bras Frutic 36:294-304. https://doi.org/10.1590/0100-2945-410/13

40. Souza KO, Moura CFH, Lopes MMDA, Rabelo MC, Miranda MRA (2017) Quality of acerola (Malpighia emarginata) treated with gibberelic acid and stored under refrigeration. Rev Bras Frutic 39:e574. https://doi.org/10.1590/0100-29452017

41. Souza FF (2015) Acerola. Cadernos do Semiárido: Riquezas e Oportunidades 4:26-27.

42. Strohecker R, Henning HM (1967) Analisis de vitaminas: métodos comprobados. Paz Montal. ed. Paz Montalvo, Madrid.

43. Vendramini AL, Trugo LC (2000) Chemical composition of acerola fruit (Malpighia punicifolia L.) at three stages of maturity. Food Chem 71:195-198. https://doi.org/10.1016/S0308-8146(00)00152-7

\section{Figures}

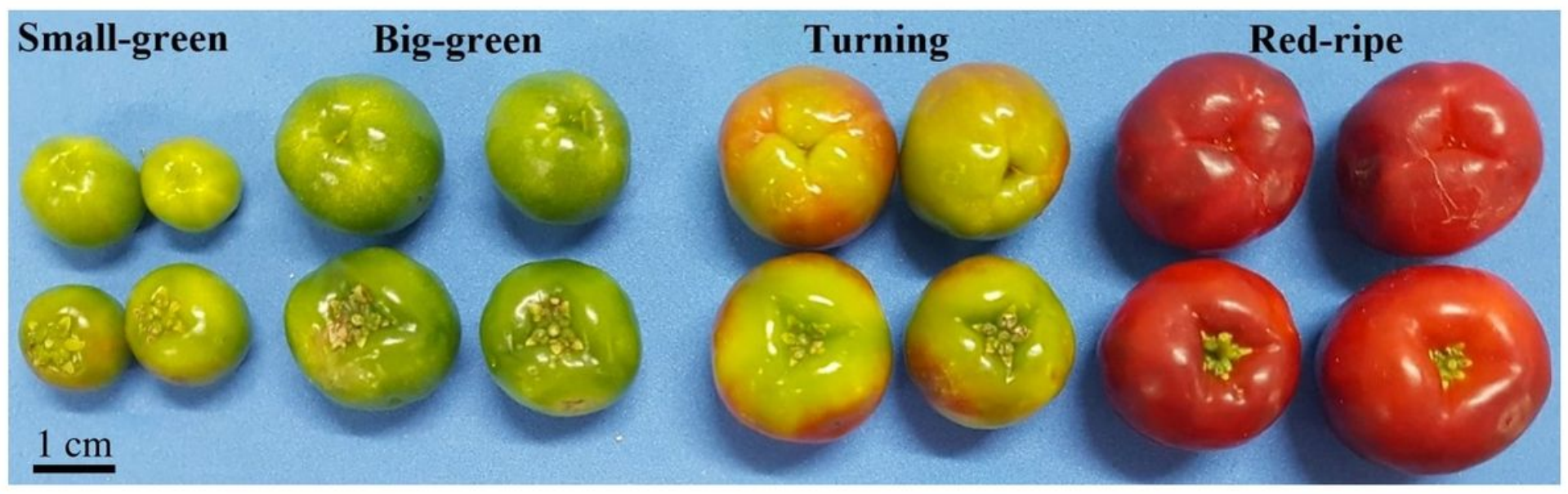

\section{Figure 1}

Acerola fruit at the maturity stages small-green, big-green, turning and red-ripe. 

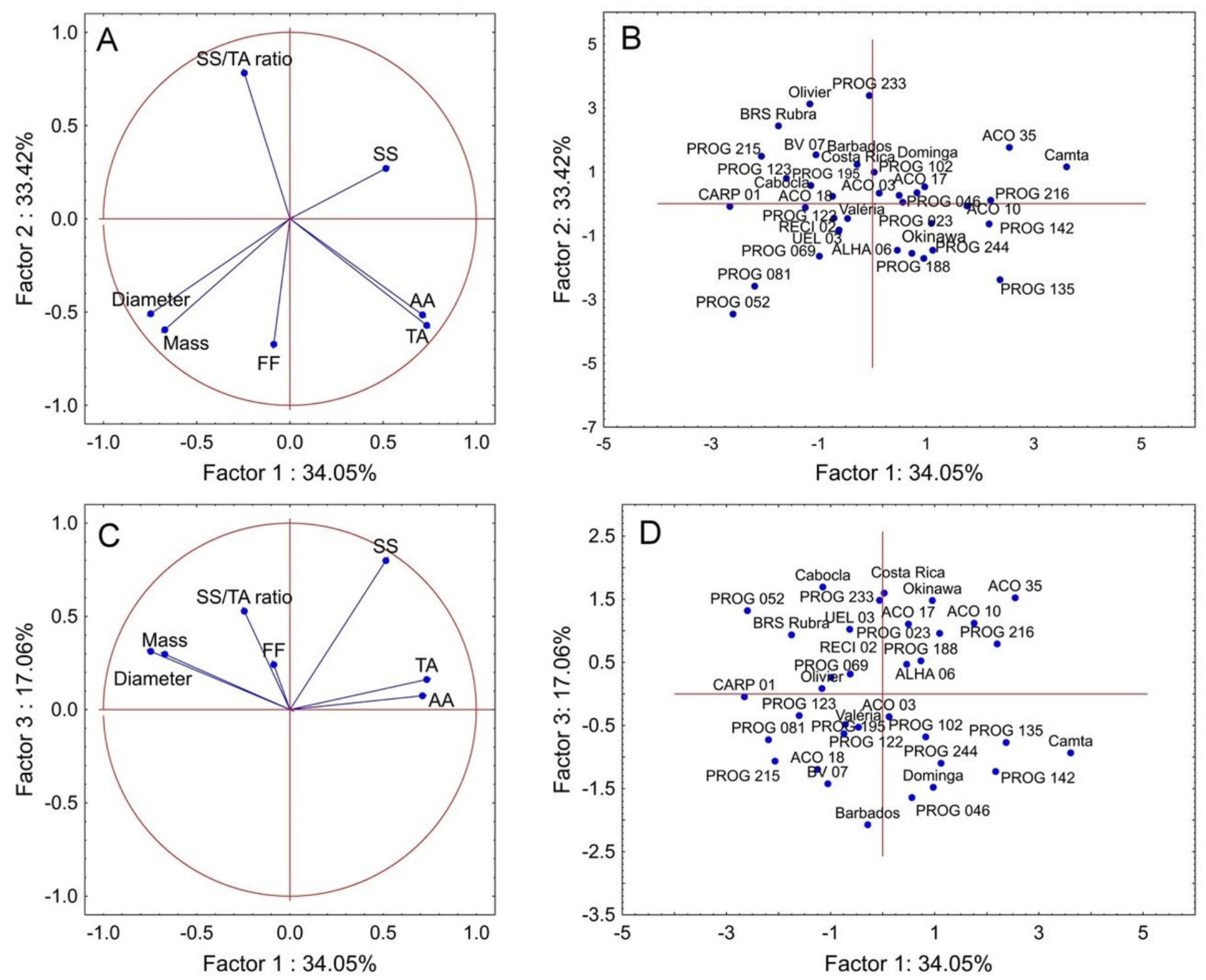

Figure 2

Principal component analysis for 35 acerola genotypes harvested at the maturity stage red-ripe in 2019, São Francisco Valley, Petrolina, PE, Brazil. 

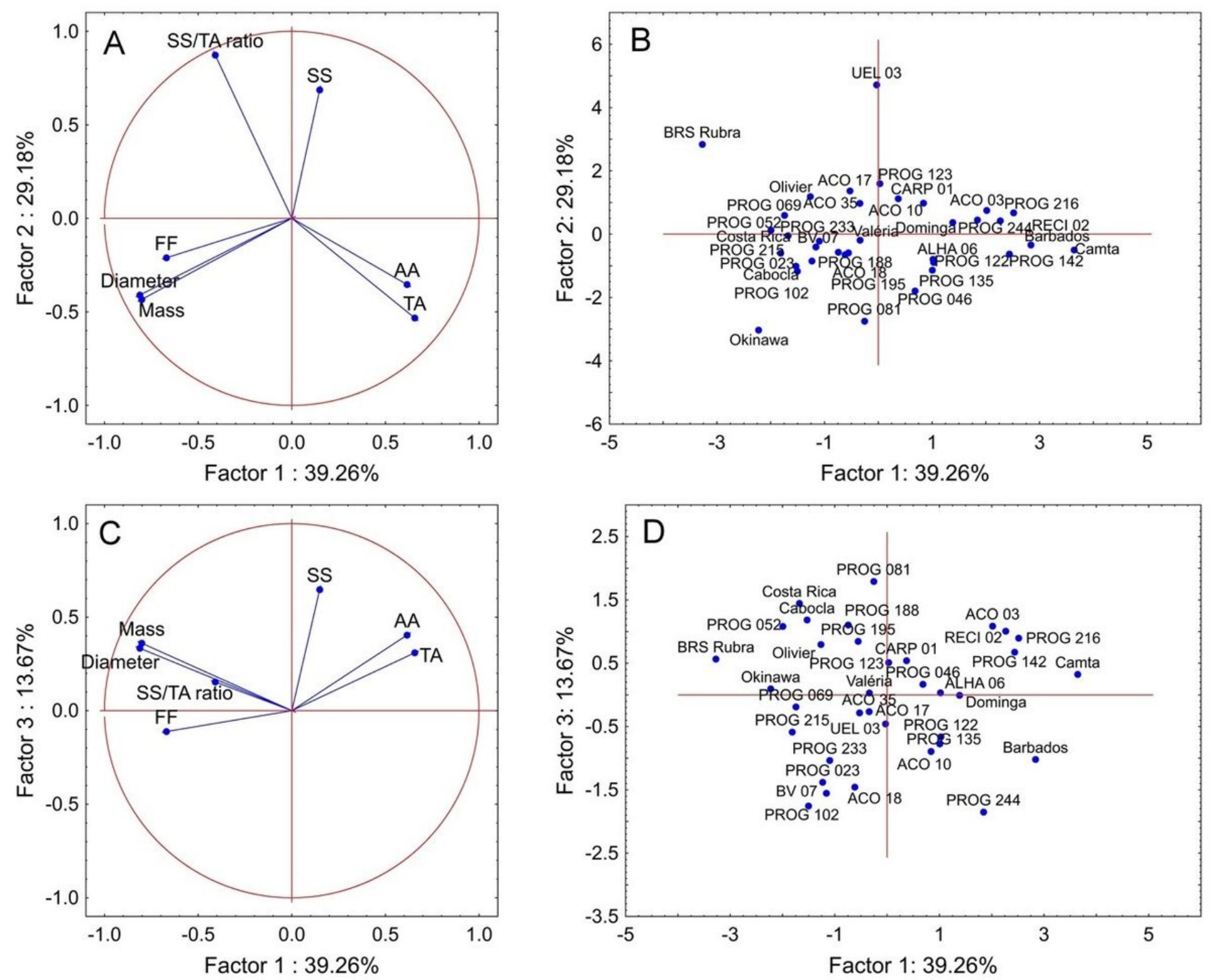

Figure 3

Principal component analysis for 35 acerola genotypes harvested at the maturity stage red-ripe in 2020, São Francisco Valley, Petrolina, PE, Brazil.

\section{Supplementary Files}

This is a list of supplementary files associated with this preprint. Click to download.

- SupplementaryTableS1.docx 